\title{
๖The Route to Raindrop Formation in a Shallow Cumulus Cloud Simulated by a Lagrangian Cloud Model
}

\author{
FABIAN HOFFMANN \\ Institute of Meteorology and Climatology, Leibniz Universität Hannover, Hannover, Germany \\ YIGN NOH \\ Department of Atmospheric Sciences, Yonsei University, Seoul, South Korea \\ SIEGFRIED RAASCH \\ Institute of Meteorology and Climatology, Leibniz Universität Hannover, Hannover, Germany
}

(Manuscript received 26 July 2016, in final form 4 April 2017)

\begin{abstract}
The mechanism of raindrop formation in a shallow cumulus cloud is investigated using a Lagrangian cloud model (LCM). The analysis is focused on how and under which conditions a cloud droplet grows to a raindrop by tracking the history of individual Lagrangian droplets. It is found that the rapid collisional growth, leading to raindrop formation, is triggered when single droplets with a radius of $20 \mu \mathrm{m}$ appear in the region near the cloud top, characterized by large liquid water content, strong turbulence, large mean droplet size, broad drop size distribution (DSD), and high supersaturations. Raindrop formation easily occurs when turbulenceinduced collision enhancement (TICE) is considered, with or without any extra broadening of the DSD by another mechanism (such as entrainment and mixing). In contrast, when TICE is not considered, raindrop formation is severely delayed if no other broadening mechanism is active. The reason for the difference is clarified by the additional analysis of idealized box simulations of the collisional growth process for different DSDs in varied turbulent environments. It is found that TICE does not accelerate the timing of the raindrop formation for individual droplets, but it enhances the collisional growth rate significantly afterward by providing a greater number of large droplets for collision. Higher droplet concentrations increase the time for raindrop formation and decrease precipitation but intensify the effect of TICE.
\end{abstract}

\section{Introduction}

Raindrop formation in warm clouds is a key question in cloud physics, which has been investigated extensively (e.g., Beard and Ochs 1993; Devenish et al. 2012; Grabowski and Wang 2013). Nonetheless, many critical questions still remain unanswered with regard to the mechanisms leading to raindrop formation. It has been difficult in particular to explain the growth of cloud droplets in the radius range of $15-40 \mu \mathrm{m}$ for which neither diffusional (or condensational) growth nor growth by collision and coalescence is effective: the so-called

๑ Denotes content that is immediately available upon publication as open access.

Corresponding author: Yign Noh, noh@yonsei.ac.kr condensation-coalescence bottleneck. Several mechanisms have been proposed to overcome this bottleneck, such as the broadening of the drop size distribution (DSD), turbulence-induced collision enhancement (TICE), and the presence of giant aerosol particles. However, it is not yet clearly understood under which conditions these processes contribute to raindrop formation.

All mechanisms for the initiation of rain, as mentioned above, propose methods to increase the collection kernel $K$, which determines the collection rate of two droplets in a unit volume. Traditionally, the gravitational collection kernel between two droplets with the radii $R$ and $r$ is used as

$$
K(R, r)=\pi(R+r)^{2}|v(R)-v(r)| E(R, r),
$$

where $v$ is the terminal fall velocity of a droplet and $E$ is the collection efficiency. To initiate rain, $K$ must become sufficiently large. 
As a mechanism to increase $K$, we can consider the fact that droplets may experience different histories of supersaturation in the turbulent environment of a cloud, and the strength of diffusional growth differs accordingly. Shear and evaporative cooling following the entrainment of dry air produces strong turbulence in cumulus clouds (e.g., Shaw 2003). The mixing of these droplets can lead to a broadening of the DSD (Cooper 1989; Blyth 1993; Lasher-Trapp et al. 2005), which increases $K$ by increasing the difference of terminal velocities in (1). The variability of supersaturation is caused by fluctuations in the concentration, size, and vertical velocity of droplets and the entrainment of environmental dry air (e.g., Politovich and Cooper 1988). In-cloud nucleation can also contribute to the broadening of DSD (Pinsky and Khain 2002), although it might play a minor role in the highly diluted shallow cumulus clouds as studied here.

Many recent studies have investigated the impact of small-scale turbulence on $K$ (e.g., Pinsky and Khain 2002; Ayala et al. 2008; Franklin 2008; Wang and Grabowski 2009). TICE increases $K$ by intensifying the relative velocity of droplets, causing local clustering of droplets, and the fortification of the collision efficiency. More sophisticated formulations of $K$ have been developed that take into account TICE, usually as a function of the dissipation rate $\varepsilon$. Wang and Grabowski (2009) showed that TICE can reduce the rain initiation time by $15 \%-40 \%$.

One can also expect from (1) that the presence of large particles increases $K$ simply by having a high terminal velocity. Accordingly, giant aerosols, which can be a part of the natural aerosol size distribution, are suggested to initiate rain if they are present in a cloud (Johnson 1982; Lasher-Trapp et al. 2001; Jensen and Lee 2008).

Probably the most appropriate way to understand the mechanism of raindrop formation is to follow the growth of individual Lagrangian droplets and to investigate how and under which conditions they grow to raindrops. For this purpose, Lasher-Trapp et al. (2005) and Cooper et al. (2013) calculated the trajectories of fluid parcels with explicit microphysics of condensation and collision-coalescence in the flow fields of a large-eddy simulation (LES) with a simple bulk parameterization of cloud microphysics. In this method, however, there was no direct feedback of the analyzed parcels to the underlying dynamical model.

Recently, Lagrangian cloud models (LCMs) have been developed in which the cloud microphysics of Lagrangian droplets and cloud dynamics are two-way coupled (e.g., Andrejczuk et al. 2008, 2010; Shima et al. 2009; Sölch and Kärcher 2010; Riechelmann et al. 2012;
Naumann and Seifert 2015). In these models, the flow field is simulated by an LES model, and the droplets are represented by Lagrangian particles, which experience microphysical processes such as condensation and evaporation, resulting in latent heating/ cooling and changes to water vapor and buoyancy (due to water loading of the drops), affecting the cloud dynamics.

A recently developed LCM is used for this study to clarify the mechanism of raindrop formation in a shallow cumulus cloud (Riechelmann et al. 2012; Lee et al. 2014; Hoffmann et al. 2015). For the present simulation, the applied LCM has been improved, especially the collision algorithm, which will be discussed in the next section. We also utilize the capability of the LCM that allows us to investigate the formation of raindrops directly by tracking the history of individual Lagrangian droplets.

We will focus on the respective roles of two effects upon raindrop formation: the broadening of DSDs and TICE. For the investigation of TICE effects, we perform the LCM with two different collection kernels by either including the effects of TICE or neglecting them. For the investigation of the effect of the DSD broadening, the results are compared with a simulation in which the diffusional growth is calculated by an adiabatic parcel model, which inhibits the broadening of DSD by entrainment and mixing, for example. Simulations are also carried out with different initial cloud condensation nuclei $(\mathrm{CCN})$ concentrations. Finally, we clarify the respective roles of the broadening of DSD and TICE by carrying out idealized box simulations, in which only collisional growth is calculated for different initial DSDs and turbulence intensities.

\section{Model and simulation setup}

The LCM used in this study is coupled to Parallelized Large-Eddy Simulation Model (PALM; Raasch and Schröter 2001; Maronga et al. 2015). The LES model solves the nonhydrostatic incompressible Boussinesqapproximated Navier-Stokes equations and equations for water vapor mixing ratio, potential temperature, and subgrid-scale turbulent kinetic energy. The LCM calculates the motion and microphysics of Lagrangian droplets. One can refer to Riechelmann et al. (2012) for the original description of this model. For the present study, the model has been improved in various aspects, including a refined collection algorithm. The basic framework of the model is described below.

To handle the extremely large number of droplets in a cloud, the concept of a superdroplet is introduced 
(Shima et al. 2009). Each superdroplet represents a large number of real droplets of identical features (e.g., their radius). The number of real droplets belonging to a superdroplet of radius $r_{n}$ is called the weighting factor $A_{n}$, and the total mass of a superdroplet $M_{n}$ is then calculated by

$$
M_{n}=A_{n} \frac{4}{3} \pi \rho_{l} r_{n}^{3}
$$

where $\rho_{l}$ is the density of liquid water. In the present model, $A_{n}$ differs for each superdroplet and changes with time as a result of collision and coalescence. The liquid water mixing ratio $q_{l}$ for a given LES grid box of the volume $\Delta V$ is then calculated by

$$
q_{l}=\frac{1}{\rho_{0} \Delta V} \sum_{n=1}^{N_{p}} M_{n},
$$

where $\rho_{0}$ is the density of dry air and $N_{p}$ is the number of superdroplets in that grid box.

The liquid water mixing ratio is considered within the virtual potential temperature,

$$
\theta_{v}=\theta\left[1+\left(\frac{R_{v}}{R_{a}}-1\right) q-q_{l}\right],
$$

to account for the drag of the droplets affecting the buoyancy in the LES (e.g., Bannon 2002):

$$
\left(\frac{d w}{d t}\right)_{\text {buoy }}=g \frac{\theta_{v}-\left\langle\theta_{v}\right\rangle}{\left\langle\theta_{v}\right\rangle} .
$$

Here, $\theta$ is the potential temperature; $R_{v}$ and $R_{a}$ are the individual gas constants of water vapor and dry air, respectively; $q$ is the water vapor mixing ratio; $w$ is the vertical velocity; $g$ is the acceleration by gravity; and angle brackets denote a horizontal average.

\section{a. Advection and sedimentation}

The velocity of each superdroplet is determined by

$$
U_{i}=u_{i}+\tilde{u}_{i}-\delta_{i 3} v(r) .
$$

The LES resolved-scale velocity at the particle's location $u_{i}$ is determined from a linear interpolation of the velocities at the eight adjacent grid points of each particle. A stochastic turbulent velocity component $\widetilde{u}_{i}$ is computed from the LES subgrid-scale turbulent kinetic energy, following Sölch and Kärcher (2010), which was absent in the old version of the model. The terminal velocity $v(r)$ is given by an empirical relationship depending on the droplet radius $r$ (Rogers et al. 1993):

$$
v(r)= \begin{cases}a_{1} r\left[1-\exp \left(-b_{1} r\right)\right], & \text { for } r \leq r_{0} \\ a_{2}-a_{3} \exp \left(-b_{2} r\right), & \text { for } r>r_{0},\end{cases}
$$

with $r_{0}=372.5 \mu \mathrm{m}, a_{1}=8000 \mathrm{~s}^{-1}, a_{2}=9.65 \mathrm{~m} \mathrm{~s}^{-1}, a_{3}=$ $10.43 \mathrm{~m} \mathrm{~s}^{-1}, b_{1}=24000 \mathrm{~m}^{-1}$, and $b_{2}=1200 \mathrm{~m}^{-1}$.

\section{b. Diffusional growth and the release/depletion of water vapor and heat}

The diffusional growth of each superdroplet is calculated from

$$
r_{n} \frac{d r_{n}}{d t}=\frac{S}{F_{k}+F_{D}} f\left(r_{n}\right),
$$

where $S$ is the supersaturation. The two coefficients in the denominator are given by $F_{k}=$ $\left[L_{v} /\left(R_{v} T\right)-1\right] L_{v} \rho_{l} /(T k)$ and $F_{D}=\rho_{l} R_{v} T /\left(D_{v} e_{s}\right)$, where $k$ is the thermal conductivity of air, $L_{v}$ is the latent heat required to convert liquid to vapor, $D_{v}$ is the molecular diffusivity of water vapor in air, and $e_{s}$ is the vapor pressure at saturation. The term $f\left(r_{n}\right)$ describes the increased evaporation of falling droplets: the so-called ventilation effect. Its parameterization is primarily based on the droplet radius $r_{n}$ (Rogers and Yau 1989).

The temporal change of $q_{l}$ due to condensation/ evaporation is then calculated as

$$
\left(\frac{d q_{l}}{d t}\right)_{\text {cond }}=\frac{1}{\rho_{0} \Delta V} \sum_{n=1}^{N_{P}} A_{n} \frac{4}{3} \pi \rho_{1} \frac{d r_{n}^{3}}{d t}
$$

and it determines the sink/source for water vapor mixing ratio $q$ and potential temperature $\theta$ in the LES model as

$$
\left(\frac{d q}{d t}\right)_{\text {cond }}=-\left(\frac{d q_{l}}{d t}\right)_{\text {cond }}
$$

and

$$
\left(\frac{d \theta}{d t}\right)_{\text {cond }}=\frac{L_{v}}{\Pi c_{p}} \times\left(\frac{d q_{l}}{d t}\right)_{\text {cond }},
$$

respectively, where $\Pi$ is the Exner function and $c_{p}$ is the specific heat capacity of air at constant pressure.

For the calculation of the supersaturation $S$ in (8), the value of the LES grid box, in which the superdroplet is currently located, is used instead of a linearly interpolated value as done in the previous versions of our LCM. This is necessary to maintain consistency with the sink/source terms for water vapor mixing ratio $q$ and potential temperature $\theta$ due to condensation/evaporation in the LES model, which are calculated by the diffusional growth of all superdroplets inside a grid box without 
considering their exact locations, as shown in (9) [see also Hoffmann (2016)].

\section{c. Collisional growth}

To calculate the droplet growth by collisioncoalescence, a statistical approach is used in which the growth of a superdroplet is calculated from the droplet spectrum resulting from all superdroplets currently located in the same LES grid box. The collisional growth is then described in terms of the modification of the weighting factor $A_{n}$ and the total mass $M_{n}$ of each superdroplet, which also results in the modification of the droplet radius $r_{n}$. While maintaining this general concept, we improved the collision algorithm for the present work by modifying the old collision algorithm (Riechelmann et al. 2015) with ideas of Shima et al. (2009) and Sölch and Kärcher (2010), as discussed below.

The collision and subsequent coalescence of a superdroplet pair with $A_{n}>A_{m}$ is realized as the collection by $A_{m}$ droplets of the superdroplet $n$. The total mass of these collected droplets, $A_{m} M_{n} / A_{n}$, is added to the droplets of superdroplet $m$, increasing $M_{m}$. Note that $A_{m}$ remains unchanged, but $r_{m}$ is increased after the collection. On the other hand, $r_{n}$ of the collected superdroplet remains unchanged, but $M_{n}$ and $A_{n}$ decrease. In addition, so-called internal collections are consideredthat is, collections of droplets belonging to the same superdroplet. These interactions do not change $M_{n}$ but they decrease $A_{n}$ and accordingly increase $r_{n}$. This yields the following description of the temporal change of $A_{n}$ (assuming that the particles are sorted that $A_{n}>A_{m}$ for $m>n)$ :

$$
\begin{aligned}
\frac{d A_{n}}{d t} \delta t= & -\frac{1}{2}\left(A_{n}-1\right) P\left[K\left(r_{n}, r_{n}\right) A_{n} \delta t / \Delta V\right] \\
& -\sum_{m=n+1}^{N_{p}} A_{m} P\left[K\left(r_{m}, r_{n}\right) A_{n} \delta t / \Delta V\right] .
\end{aligned}
$$

The first term on the right-hand side describes the decrease of the droplet number due to internal collections; the second term denotes the loss of droplets due to coalescence with droplets represented by a superdroplet with a smaller weighting factor. The probabilistic binary function $P[\varphi]$ determines if a collection takes place based on its argument, the collection probability $\varphi$ :

$$
P[\varphi]=\left\{\begin{array}{lll}
0 & \text { if } & \varphi \leq \xi, \\
1 & \text { if } & \varphi>\xi,
\end{array}\right.
$$

where $\xi$ is a random number uniformly chosen from the interval $[0,1]$. The change of the total mass of a superdroplet is then calculated by

$$
\begin{aligned}
\frac{d M_{n}}{d t} \delta t= & \sum_{m=1}^{n-1} A_{n} \frac{M_{m}}{A_{m}} P\left[K\left(r_{n}, r_{m}\right) A_{m} \delta t / \Delta V\right] \\
& -\sum_{m=n+1}^{N_{p}} A_{m} \frac{M_{n}}{A_{n}} P\left[K\left(r_{m}, r_{n}\right) A_{n} \delta t / \Delta V\right] .
\end{aligned}
$$

The first term on the right-hand side denotes the gain of mass due to the collection with superdroplets of a larger weighting factor; the second term denotes the loss of mass due to the collection of droplets belonging to a superdroplet with a smaller weighting factor.

The new collision algorithm is different from the old collision algorithm in two important aspects. They rectify the problem of the old algorithm, associated with the difficulty of the correct representation of the stochastic collisional growth process, which produces a small number of very large droplets.

First, the superdroplet with the smaller weighting factor now collects droplets from the superdroplet with the larger weighting factor. In the old algorithm, the superdroplet with the larger radius collected droplets from the superdroplet with the smaller radius. Consider the case of the largest superdroplet. The weighting factor of the largest superdroplet did not decrease in the old algorithm since it grew by collecting mass from smaller superdroplets without being collected at any time. Accordingly, the largest superdroplet always represented a large number of real droplets, and the collected mass from smaller superdroplets was equally distributed over these. As a result, the radius growth of the largest superdroplet was too slow. In the new collision algorithm, however, the largest superdroplets tend to have the smallest weighting factors, because they are more likely to collect other superdroplets.

Second, the collection is now treated as a binary ( 0 or 1) process, in which either all droplets of the collecting superdroplet coalesce with the same number of droplets from the collected superdroplet or none do [see (13)]. In this way the radius growth of a superdroplet by collision and coalescence resembles the growth of two real droplets coalescing; that is, one droplet collects a complete other droplet, contrary to a fraction of it as in the old algorithm with continuous collection probability. As the number of superdroplets becomes large, the new algorithm tends to produce the size distribution of superdroplets corresponding to the size distribution of real droplets, while the old algorithm tended to produce a more uniform growth of superdroplets. In that sense, the old and new algorithms approximate either continuous or stochastic collisional growth, respectively (Telford 1955).

Both changes are already in use in the collision algorithms by Shima et al. (2009) and Sölch and Kärcher (2010). 

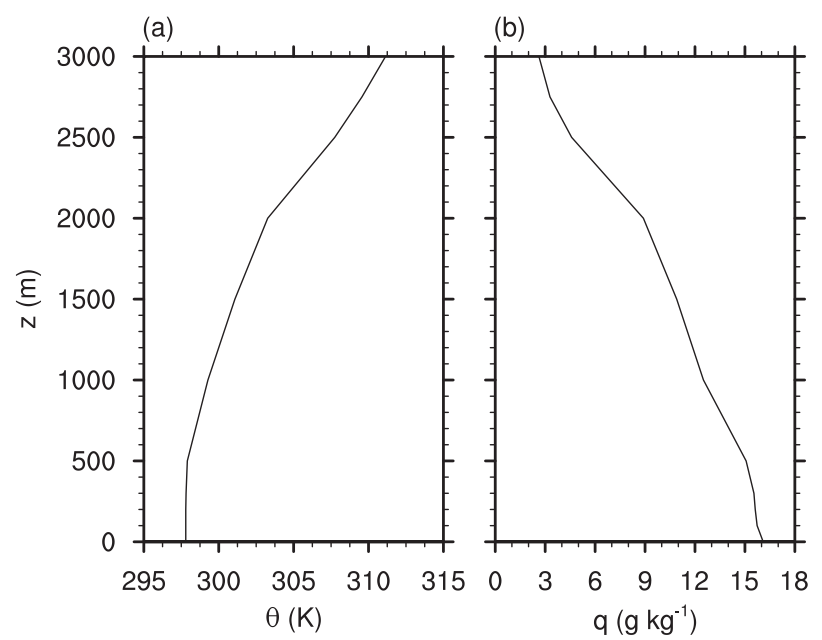

FIG. 1. Initial profiles of (a) potential temperature $\theta$ and (b) water vapor mixing ratio $q$.

One can refer to Unterstrasser et al. (2017) for more details on this so-called all-or-nothing collection algorithm and a comparison with other Lagrangian collection algorithms, including our old algorithm, and a spectral-bin model.

\section{d. Simulation setup}

A shallow cumulus cloud is triggered by a twodimensional rising bubble of warm air, which is homogeneous in the third spatial direction. The bubble is prescribed by an initial potential temperature difference $\theta^{*}$ given by

$$
\theta^{*}=\theta_{0}^{*} \exp \left\{-\frac{1}{2}\left[\left(\frac{y-y_{c}}{a_{y}}\right)^{2}+\left(\frac{z-z_{c}}{a_{z}}\right)^{2}\right]\right\},
$$

where $y_{c}=1920 \mathrm{~m}$ and $z_{c}=150 \mathrm{~m}$ mark the center of the bubble, $a_{y}=200 \mathrm{~m}$ and $a_{z}=170 \mathrm{~m}$ the radius of the bubble, and $\theta_{0}^{*}=0.4 \mathrm{~K}$ the maximum temperature difference. The model domain is $1920 \times 5760 \times 3840 \mathrm{~m}^{3}$ along the $x, y$, and $z$ directions with an isotropic grid spacing of $20 \mathrm{~m}$. Periodic boundary conditions are applied laterally, and Dirichlet and Neumann boundary conditions are applied at the bottom and top, respectively. The initial profiles of potential temperature and water vapor mixing ratio are derived from the LES intercomparison of shallow cumulus convection by vanZanten et al. (2011) and are shown in Fig. 1. They represent the average thermodynamic state of a cumulus-topped boundary layer as observed during the Rain in Cumulus over the Ocean (RICO) field campaign (Rauber et al. 2007). Furthermore, no background winds, no large-scale subsidence, and no surface fluxes are applied. Note that the cloud motion is mainly driven by the latent heating of condensation, once the cloud reaches the lifting condensation level (LCL). Therefore, the cloud motion is not sensitive to the initial distribution of $\theta^{*}$, as long as the initial buoyancy is strong enough to reach the LCL, and the size of the initial bubble is much smaller than the cloud size.

Superdroplets are released $5 \mathrm{~min}$ after the start of the simulation randomly all over the model domain up to a height of $2800 \mathrm{~m}$, using a random generator for the spatial placement of each superdroplet. This delayed release of particles is necessary to avoid false divergence in the superdroplet field during the initial rise of the bubble of warm air, which is induced by the linear interpolation of the LES resolved-scale velocities to the particle location under strong vertical acceleration. Note that no cloud physical effects are missed owing to the delayed introduction of superdroplets because the bubble of warm air does not reach its LCL at $t=5 \mathrm{~min}$.

The average distance between superdroplets is initially $3.4 \mathrm{~m}$, yielding a total number of $7.9 \times 10^{8}$ superdroplets and about 200 superdroplets per grid box, which is on the edge of computational feasibility and larger than the superdroplet concentrations usually reported as sufficient for the correct representation of cloud physics (e.g., Riechelmann et al. 2012; Arabas and Shima 2013). Using an average value of $A_{n, \text { init }}=0.8 \times 10^{9}, 2.8 \times 10^{9}$, and $6.0 \times 10^{9}$, droplet number concentrations of approximately 20,70 , and $150 \mathrm{~cm}^{-3}$ are simulated. Unless stated otherwise, the results from the $70-\mathrm{cm}^{-3}$ simulations are used for analysis. The initial weighting factor of each particle has been perturbed by a random factor chosen uniformly from the interval $[0,2]$. This approach allows a better representation of the collisional growth process for a given number of superdroplets by improving the statistics of the largest droplets, which preferentially grow from the superdroplets with the smallest weighting factors (Unterstrasser et al. 2017). The radius of all superdroplets is initially given by $r=0.01 \mu \mathrm{m}$, and the particles are not allowed to evaporate any smaller. A time step of $\Delta t=0.2 \mathrm{~s}$ is used in both LCM and LES.

To clarify the role of cloud microphysics in raindrop formation, two simulations are carried out for each droplet concentration with different collection kernels $K$. The first simulation uses the traditional formulation of $K$ by Hall (1980), which considers only gravitational collision and coalescence, and the second simulation includes TICE by parameterizations of particle relative velocities and clustering (Ayala et al. 2008) and enhanced collision efficiencies (Wang and Grabowski 2009) to the Hall (1980) kernel. In the latter case, the enhancement of the collection kernel by turbulence is 

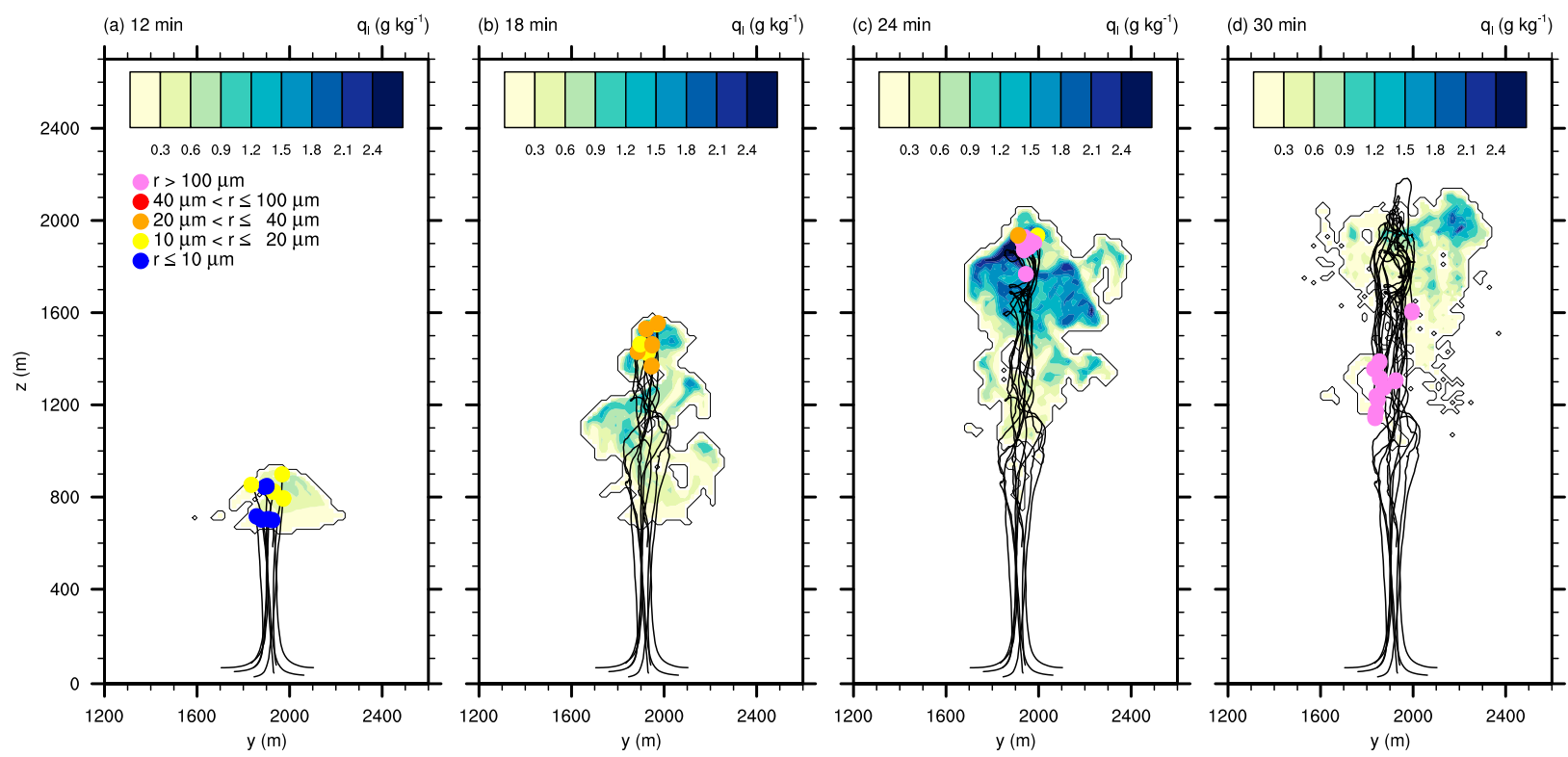

FIG. 2. Evolution of liquid water mixing ratio at a vertical cross section at $t=$ (a) 12 , (b) 18 , (c) 24 , and (d) 30 min, overlaid with 10 Lagrangian droplets that grow to the largest raindrops during the evolution of the cloud until $t=35 \mathrm{~min}$ within $\pm 50 \mathrm{~m}$ of the cross section. The color of a droplet changes according to its size (blue: $r \leq 10 \mu \mathrm{m}$; yellow: $10<r \leq 20 \mu \mathrm{m}$; orange: $20<r \leq 40 \mu \mathrm{m}$; red: $40<r \leq 100 \mu \mathrm{m}$; violet: $r>100 \mu \mathrm{m})$.

parameterized as a function of the dissipation rate $\varepsilon$, which is calculated from the LES subgrid-scale model (Riechelmann et al. 2012). The coalescence efficiency has been assumed as unity. ${ }^{1}$ These simulations are called GRAV and TURB, respectively. Furthermore, with the aim to investigate the effect of DSD broadening, additional calculations are carried out, in which the diffusional growth is calculated by an adiabatic parcel model (APM; see appendix A for a description of the APM).

\section{Results}

\section{a. Evolution of a cumulus cloud and raindrop formation}

Figure 2 shows cross sections of the liquid water mixing ratio $q_{l}$ at $t=12,18,24$, and $30 \mathrm{~min}$ in the case of TURB. Overlaid are the trajectories of 10 Lagrangian droplets that grow to the largest raindrops during the evolution of the cloud $(t<35 \mathrm{~min})$ within $\pm 50 \mathrm{~m}$ of the cross section (herein superdroplets are called droplets for convenience). The cloud base height is about $600 \mathrm{~m}$ with an absolute temperature of $293.3 \mathrm{~K}$, and

\footnotetext{
${ }^{1}$ The Weber number has been calculated for all collections and is generally smaller than 0.1 during the initial collisions and the triggering of the rapid collisional growth, which validates the assumption of a unity coalescence efficiency (e.g., Beard and Ochs 1984).
}

the maximum vertical velocity rarely exceeds $4 \mathrm{~m} \mathrm{~s}^{-1}$ (not shown).

During the initial updraft stage $(t=12 \mathrm{~min})$, droplets already exist inside the cloud. As the cloud is developed further $(t=18 \mathrm{~min})$, most droplets tend to be located in the region with large $q_{l}$ near the cloud top, and raindrops $(r>40 \mu \mathrm{m})$ appear there at $t=24 \mathrm{~min}$. Here we refer to raindrops for droplets of $r>40 \mu \mathrm{m}$, similar to many bulk cloud microphysical models, which represents the transition from cloud droplets to raindrops induced by the dominance of collisional growth (e.g., Kessler 1969; Berry and Reinhardt 1974; Kogan 2013). Once raindrops are formed $(t>24 \mathrm{~min})$, they fall through the cloud with radii up to $500 \mu \mathrm{m}$ (not visible from Fig. 2). Formation of large drops near the cloud top of shallow cumuli is in agreement with the observation of shallow cumuli during RICO (Small and Chuang 2008). For example, Fig. 3 in Small and Chuang (2008) showed that drops larger than $r=100 \mu \mathrm{m}$ only appear near the cloud top, although the frequency distributions of smaller droplets $(r<20 \mu \mathrm{m})$ have the similar shape near the cloud top and at the midcloud level.

Figure 3 shows the cross sections of $r_{\text {eff }}, \sigma_{r}, \varepsilon$, and $S$ soon after the raindrop formation $(t=24 \mathrm{~min})$ for the same cloud displayed in Fig. 2. Here $r_{\text {eff }}$ is the effective radius of droplets, and $\sigma_{r}^{2}$ is the variance of $r$ in a grid box, which represents the width of the DSD. Values of $S$ outside the cloud are not displayed to increase clarity. Large values of $q_{l}, \varepsilon$, and $S$ appear near the cloud top but 

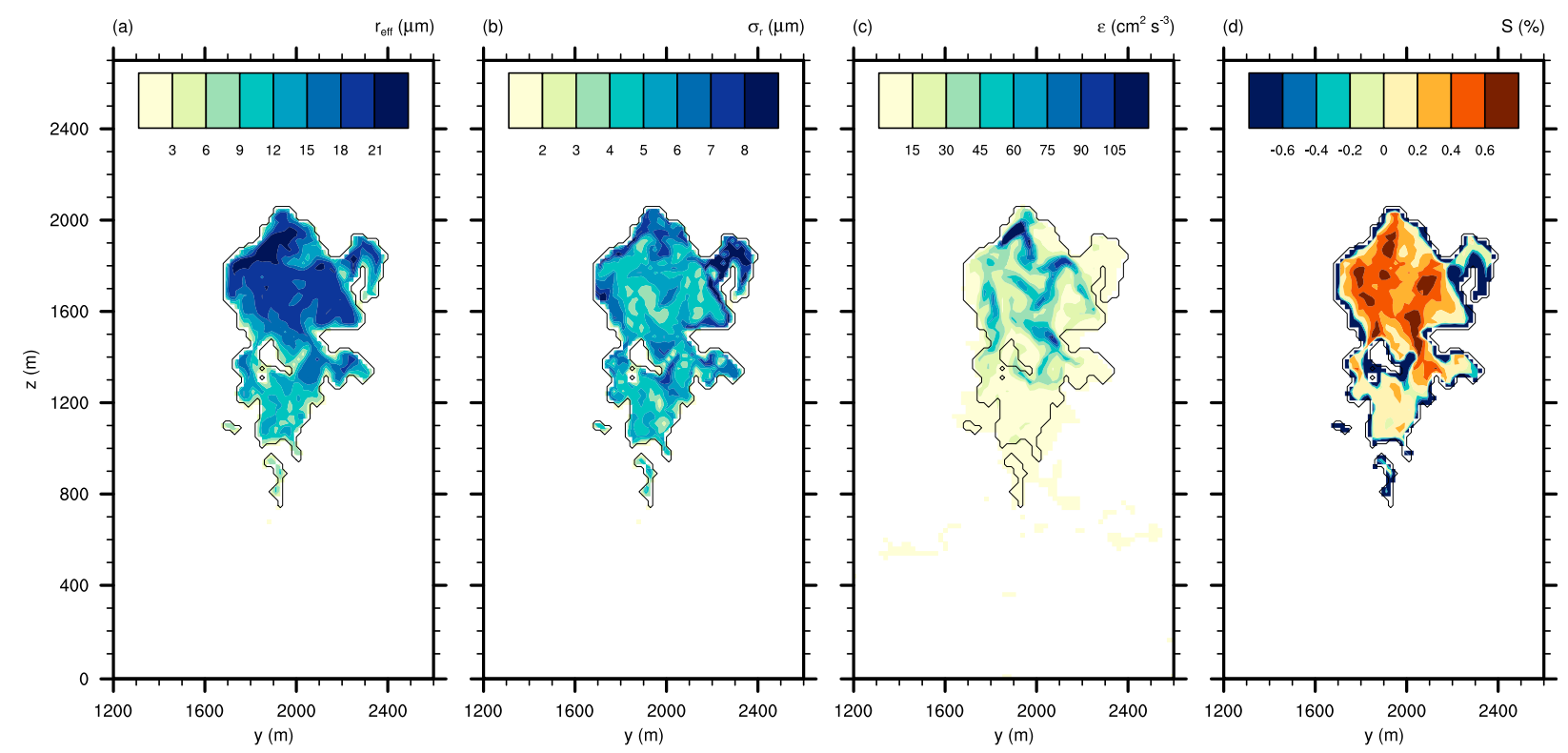

FIG. 3. Other variables at $t=24 \mathrm{~min}$ of the cross section displayed in Fig. 2 (TURB): (a) $r_{\text {eff }}$, (b) $\sigma_{r}$, (c) $\varepsilon$, and (d) $S$.

away from the cloud edge. On the other hand, large values of $\sigma_{r}$ appear near the cloud edge, indicating the broadening of DSD initiated by the entrainment of dry air and subsequent mixing. Values of $r_{\text {eff }}$ tend to increase with height. Furthermore, Figs. 2 and 3 also reveal the strong fluctuation of these values under the influence of turbulent mixing not only at the cloud edge but also in the cloud core.

To examine the variability of these quantities, we calculated the probability density functions (PDFs) of $q_{l}, r_{\mathrm{eff}}, \sigma_{r}, \sigma_{r} / r_{\mathrm{eff}}, \varepsilon$, and $S$ at $t=24 \mathrm{~min}$ from both GRAV and TURB (Fig. 4). Calculations are made for the data in the whole cloud and at the locations of potential raindrops, separately. Here we use the term potential raindrops for the Lagrangian superdroplets whose maximum size during the evolution of the cloud until $t=$ 35 min belongs to the largest 50 raindrops. Potential raindrops that enter the cloud after $t=12 \mathrm{~min}$ are filtered out in order to focus on droplets with similar entrainment times. Sensitivity to the sampling size and to the filtering is discussed in appendix B. The cloud is defined as the region where $q_{l}>1.0 \times 10^{-2} \mathrm{~g} \mathrm{~kg}^{-1}$. The distributions of $q_{l}$ and $\varepsilon$ within the cloud indicate an intermittent nature in which very large values are concentrated within small regions, while most other regions are filled with small values. The large variability of $S$ within the cloud, as observed in Figs. $3 \mathrm{~d}$ and 4e, provides the favorable condition for the broadening of DSD by droplets following different trajectories with different supersaturation histories to the point of observation, as suggested by Baker et al. (1980), Cooper (1989),
Blyth (1993), and Lasher-Trapp et al. (2005). The PDF of $\sigma_{r}$ indicates that potential raindrops exist less frequently in regions with $\sigma_{r}<3 \mu \mathrm{m}$ and more frequently in regions with $\sigma_{r}>7 \mu \mathrm{m}$, resulting in a generally larger mean value of $\sigma_{r}$ for potential raindrops. The fact that a large portion of the region within the cloud is subsaturated $(S<0)$ also reveals how much the cloud is affected by the entrainment of dry environmental air (Fig. 4e).

Figure 4 shows the strong tendency of preferential concentration of potential raindrops in the region of high $q_{l}, r_{\mathrm{eff}}, \sigma_{r}, \varepsilon$, and $S$, which is in agreement with observations (Small and Chuang 2008; Gerber et al. 2008; Arabas et al. 2009) and with other simulation results (Cooper et al. 2013; Khain et al. 2013). In particular, Small and Chuang (2008) and Arabas et al. (2009) found that the largest drops are located in the regions that have a DSD shifted to larger sizes, and in the regions that have experienced strong entrainment. It means that raindrops are likely to form in the regions where the conditions are favorable to collisional growth, such as a large liquid water mixing ratio $q_{l}$, strong turbulence $\varepsilon$, a large effective radius $r_{\text {eff }}$, a broad DSD $\sigma_{r}$, and high supersaturations $S$. Most potential raindrops are located within supersaturated regions $(S>0)$ in particular. One can also infer from Fig. $4 \mathrm{~d}$ that $\sigma_{r} / r_{\mathrm{eff}}$ is primarily about 0.6 in the whole cloud, and it is much smaller at the locations of potential raindrops (about 0.3 and 0.2 for GRAV and TURB, respectively).

Furthermore, the PDFs at the locations of potential raindrops are different between GRAV and TURB, although the PDFs sampled for the whole cloud are 

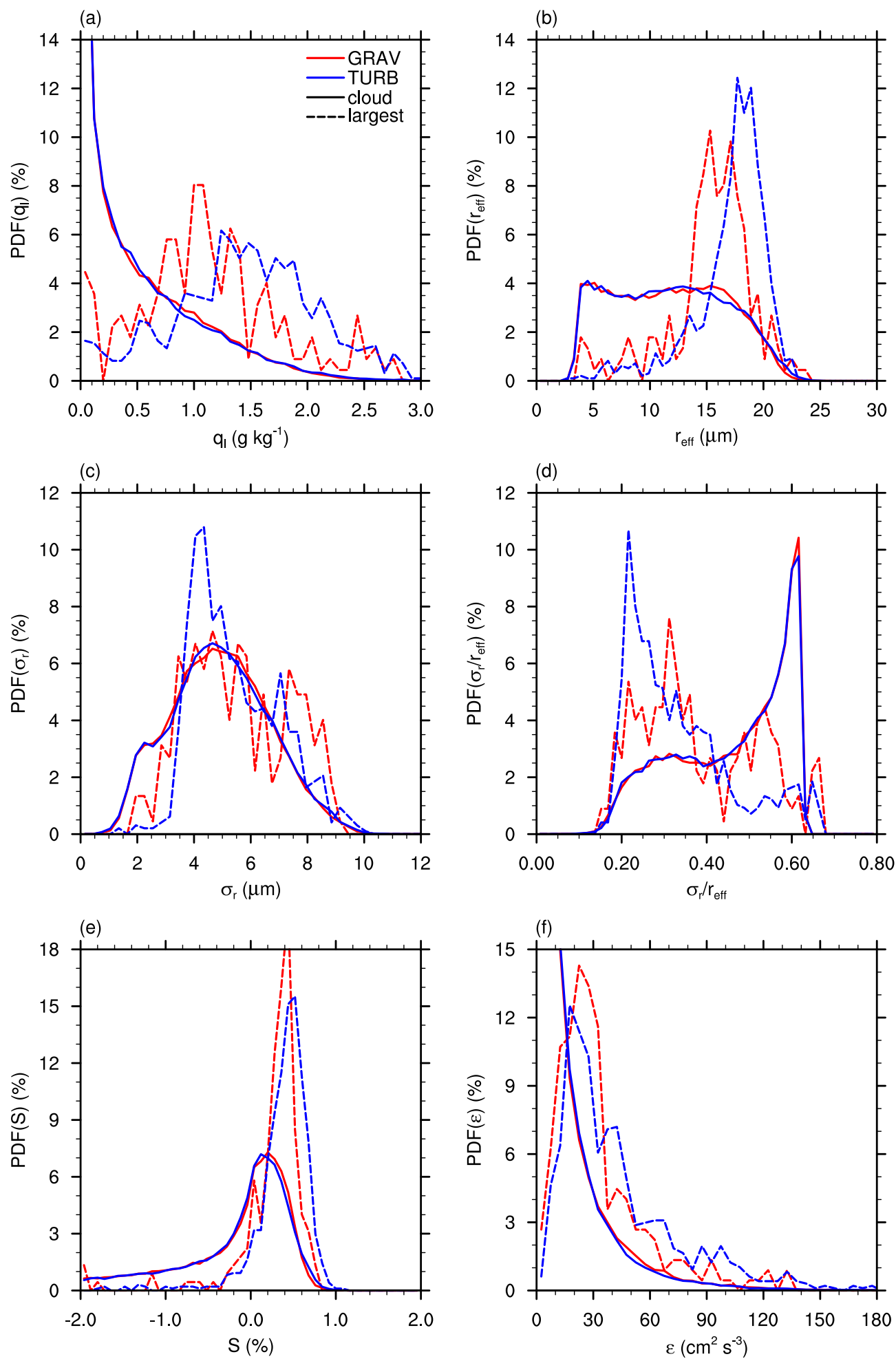

FIG. 4. PDFs of variables at $t=24 \mathrm{~min}$ in the whole cloud (solid line) and at the locations of potential raindrops (dashed line) (red: GRAV; blue: TURB): (a) $q_{l}$, (b) $r_{\text {eff }}$, (c) $\sigma_{r}$, (d) $\sigma_{r} / r_{\text {eff }}$, (e) $S$, and (f) $\varepsilon$. 
virtually the same. The tendency of raindrop formation in the regions of high $q_{l}, r_{\mathrm{eff}}, \varepsilon$, and $S$ are stronger in TURB, while the tendency of raindrop formation in the regions of high $\sigma_{r}$ is stronger in GRAV. It suggests the possibility that the broadening of DSD plays a more important role in GRAV than in TURB for the formation of raindrops, while the large values of $q_{l}, r_{\mathrm{eff}}, \varepsilon$, and $S$ play more important roles in TURB, which will be discussed further in the next section.

\section{b. The route to raindrop formation}

Figure 5 shows the time series of the ensemble average of various quantities following potential raindrops, such as $R, \sigma_{r}, q_{l}, \varepsilon, r_{\mathrm{eff}}, N, Z, d R_{C} / d t$, and $d R_{D} / d t$, for GRAV and TURB ( $R$ and $Z$ are the radius and height of potential raindrops, $N$ is the droplet concentration, and $d R_{D} / d t$ and $d R_{C} / d t$ are diffusional and collisional radius growth rates, respectively). Here it is important to note that $R, Z, d R_{D} / d t$, and $d R_{C} / d t$ represent the mean values of potential raindrops themselves, $\varepsilon$ represents the value of the grid boxes in which potential raindrops are located, and $q_{l}, \sigma_{r}, r_{\text {eff }}$, and $N$ represent the statistics derived from all superdroplets within the grid boxes in which the potential raindrops are located. Also shown are the time series from the APM for both cases of GRAV and TURB (dashed lines).

The most remarkable result is that raindrops $(r>$ $40 \mu \mathrm{m}$ ) are formed in TURB in both cases of LCM and APM, unaffected by the broadening of DSD. Note that $\sigma_{r}$ increases substantially in the LCM before the onset of the raindrop formation $(t=24 \mathrm{~min})$, while $\sigma_{r}$ remains very small in the APM. In this case, one can argue that raindrops can be formed only if $r_{\text {eff }}$ becomes sufficiently large, as suggested by Khain et al. (2013), and the broadening of DSD does not play an important role in the raindrop formation.

On the other hand, in the case of GRAV, raindrops are severely delayed in the APM, even though $q_{l}$ and $r_{\text {eff }}$ are larger than in the LCM. It indicates clearly that raindrop formation in time requires the broadening of DSD, which may be produced by entrainment and mixing, if TICE is absent. The fact that $q_{l}$ and $r_{\text {eff }}$ calculated by the LCM are smaller than the predictions by the APM also confirms the effect of entrainment (Figs. $5 \mathrm{c}$ and $5 \mathrm{e}$ ).

At about $t=21 \mathrm{~min}$, the rapid increase of $d R_{C} / d t$ appears in both GRAV and TURB, followed by the rapid increase of $R$. We can regard this time as the triggering of the rapid collisional growth $T_{C}$ leading to precipitation. The rapid collisional growth can also be identified by the decrease of $N$ after $T_{C}$ in Fig. 5 f. The decrease of $N$ from the APM for TURB becomes very large because of the stronger collection in undiluted cloud air, which is also reflected by the rapid increases of $\sigma_{r}$ (Figs. 5b and 5f). Radius $R$ grows to the size of raindrops $(r=40 \mu \mathrm{m})$ at about $t=24 \mathrm{~min}$, soon after $T_{C}$. The fact that $R$ reaches about $20 \mu \mathrm{m}$ at $t=T_{C}$ confirms the consensus in the raindrop formation process that the collisional growth can become significant only after some droplets reach a radius of $r=20 \mu \mathrm{m}$ or so (Rogers and Yau 1989). Meanwhile, $r_{\mathrm{eff}}=16 \mu \mathrm{m}$ at $t=T_{C}$, which is somewhat larger than the observational evidence that the rapid formation of raindrops in convective clouds begins when $r_{\text {eff }}$ exceeds about 11-15 $\mu \mathrm{m}$ (e.g., Freud and Rosenfeld 2012; Khain et al. 2013).

Another remarkable feature of the results is that the effect of TICE hardly affects $T_{C}$ but increases $d R_{C} / d t$ significantly afterward $(t>25 \mathrm{~min})$. Meanwhile, the larger $d R_{C} / d t$ after $T_{C}$ under the influence of TICE leads to earlier and stronger precipitation (see also Figs. $6 \mathrm{~d}$ and $8 \mathrm{a}$ ), which is consistent with previous simulations using Eulerian cloud models (Pinsky and Khain 2002; Wang and Grabowski 2009; Seifert et al. 2010; Wyszogrodzki et al. 2013; Grabowski et al. 2015).

The value of $\varepsilon$ is slightly larger in TURB, which may reflect the fact that potential raindrops are located preferentially in highly turbulent regions (see Fig. 4d). Similarly, $Z$ becomes slightly larger in TURB after $T_{C}$, probably because the regions with the maximum $\varepsilon$ are located closer to the cloud top (Fig. 3). It should be mentioned that no significant difference between GRAV and TURB exists in the vertical distributions of the cloud as expected from Fig. 4. Soon after the onset of raindrop formation, $q_{l}, \sigma_{r}, \varepsilon$, and $r_{\text {eff }}$ decrease following potential raindrops that settle gravitationally. The decrease starts earlier in TURB, in which the raindrops become larger and precipitation starts earlier, in agreement with Wyszogrodzki et al. (2013) and Grabowski et al. (2015), who reported an increased offloading of droplets when TICE is considered.

To understand the route to raindrop formation more clearly, we divide the time series of $q_{l}$ in Fig. $5 \mathrm{c}$ - that is, the liquid water mixing ratio of the grid boxes in which the tracked potential raindrops are located-according to four different droplet size ranges, which are $q_{l}(r \leq 10 \mu \mathrm{m}), q_{l}(10<r \leq 20 \mu \mathrm{m}), q_{l}(20<r \leq 40 \mu \mathrm{m})$, and $q_{l}(r>40 \mu \mathrm{m})$ displayed in Fig. 6 . The magnitude of $q_{l}(r \leq 10 \mu \mathrm{m})$ shows a rapid increase to a sharp peak near $t=11 \mathrm{~min}$ due to diffusional growth during the initial updraft and then decreases rapidly as droplets grow to the range of larger droplets $q_{l}(10<r \leq 20 \mu \mathrm{m})$. Thereafter, $q_{l}(r \leq 10 \mu \mathrm{m})$ is maintained at a certain level, reflecting the contribution from the introduction of new droplets through entrainment and the presence of other droplets having experienced strong evaporation. Figure 6 also shows that $q_{l}(10<r \leq 20 \mu \mathrm{m})$ and $q_{l}(20<r \leq 40 \mu \mathrm{m})$ appear at $t=10$ and $15 \mathrm{~min}$, 

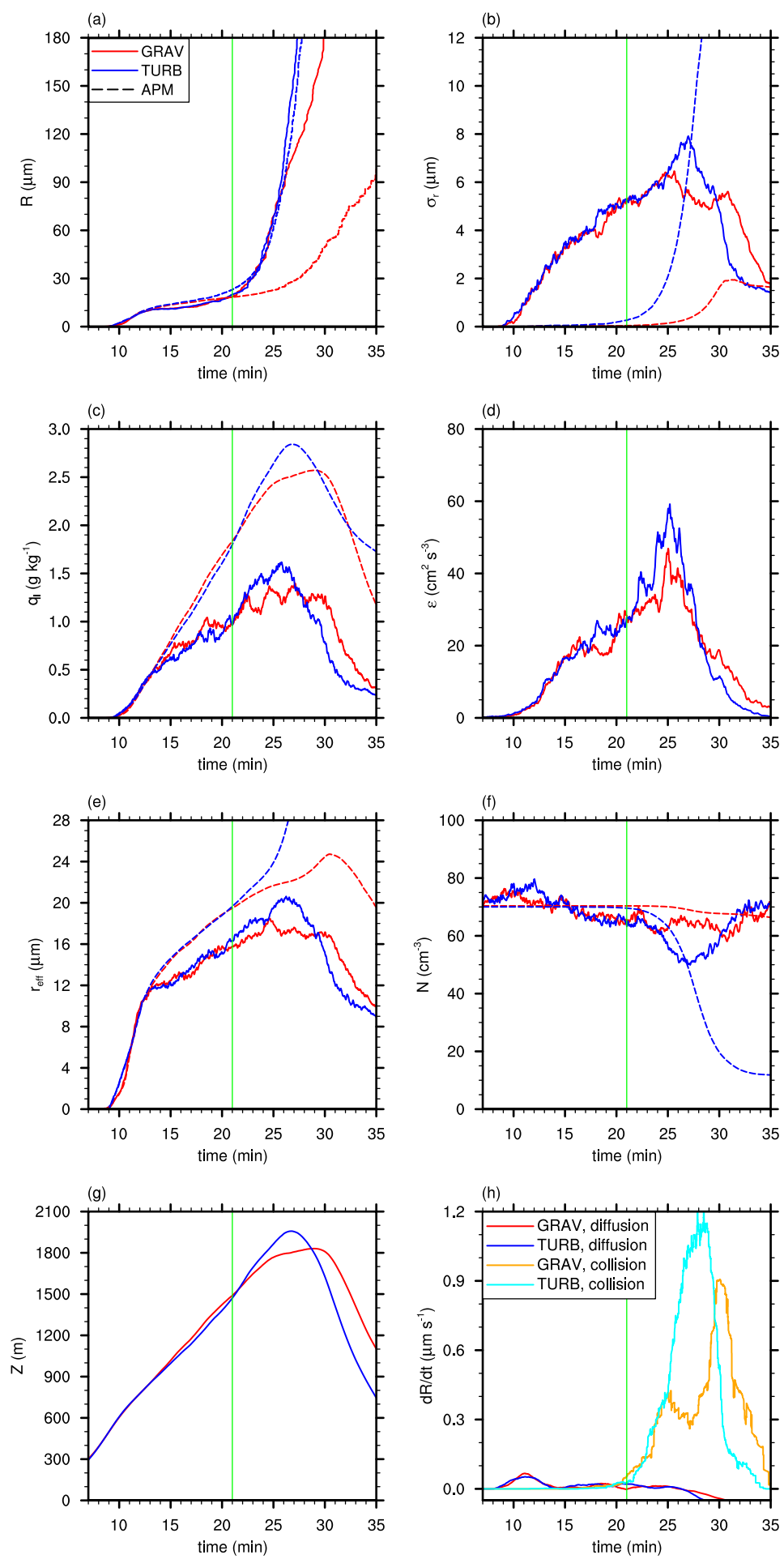

FIG. 5. Time series of the ensemble average of physical variables following potential raindrops (red: GRAV; blue: TURB; solid line: LCM; dotted line: APM; green vertical line: $t=T_{C}$ ): (a) $R$, (b) $\sigma_{r}$, (c) $q_{l}$, (d) $\varepsilon$, (e) $r_{\text {eff }}$, (f) $N$, (g) $Z$, and $(\mathrm{h}) d R_{C} / d t$ and $d R_{D} / d t$. 
(a)

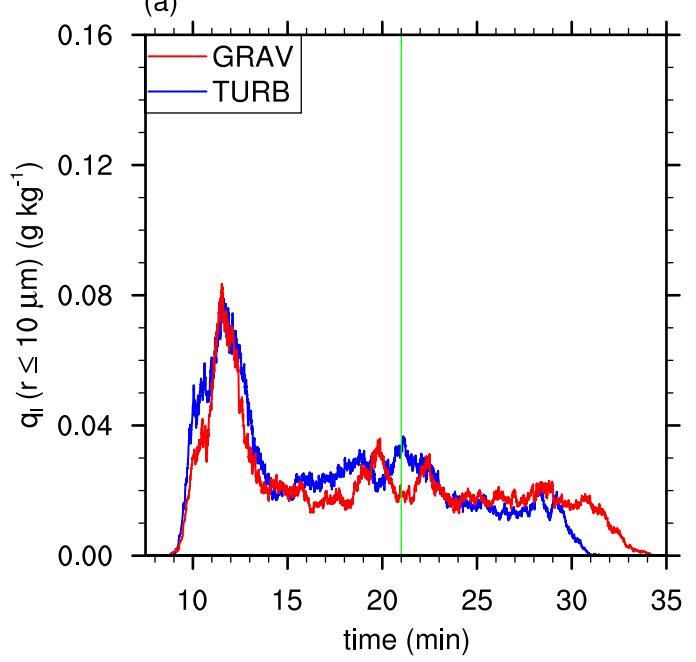

(c)

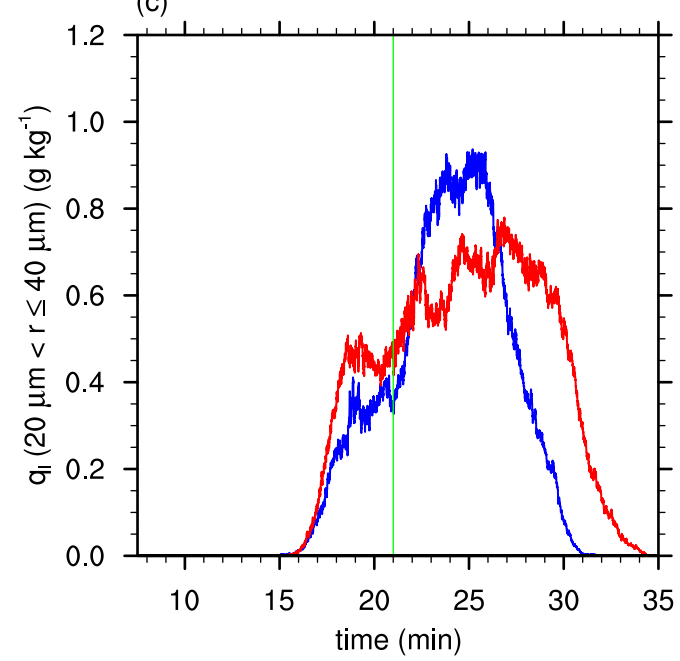

(b)

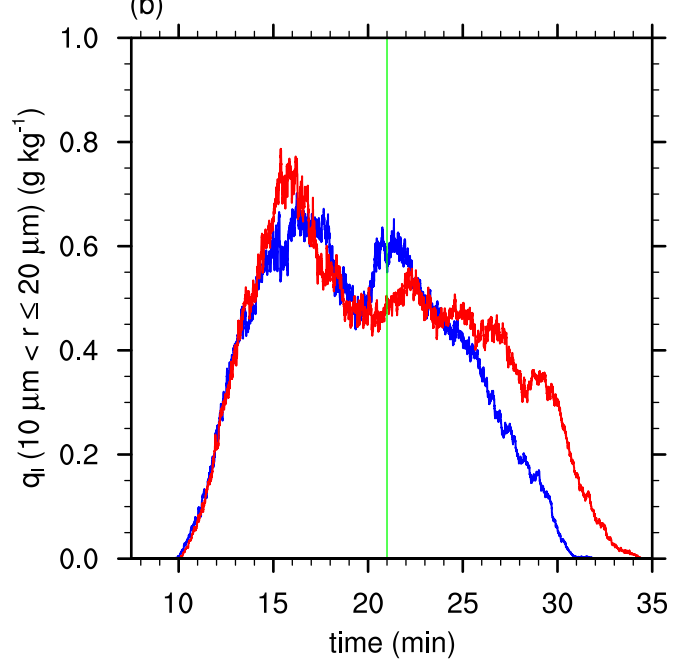

(d)

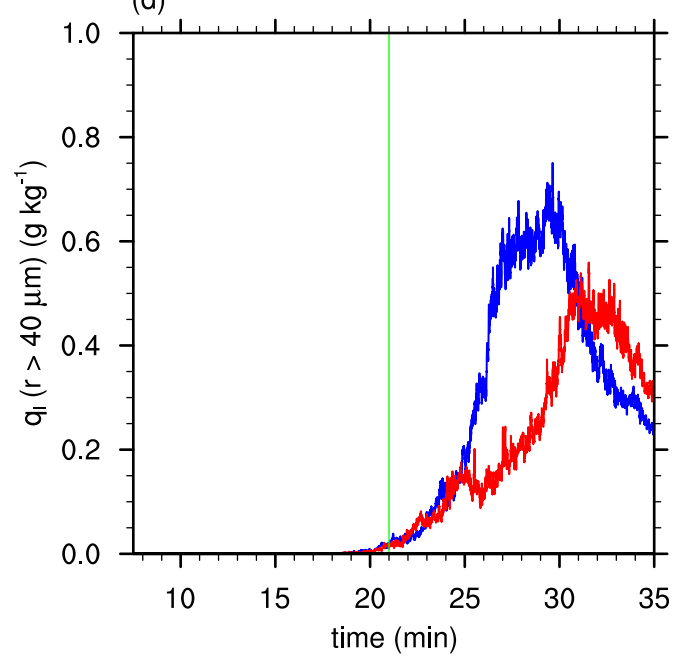

FIG. 6. Time series of the ensemble average of $q_{l}$ according to different size ranges following potential raindrops (red: GRAV; blue: TURB; green vertical lines: $t=T_{C}$ ): (a) $r \leq 10 \mu \mathrm{m}$, (b) $10<r \leq 20 \mu \mathrm{m}$, (c) $20<r \leq 40 \mu \mathrm{m}$, and (d) $r>40 \mu \mathrm{m}$.

respectively, but both start to decrease as the droplets are coalesced to larger droplets. The production of $q_{l}(r>40 \mu \mathrm{m})$ starts to appear at $t=21 \mathrm{~min}$, and it becomes much larger in TURB after $t=25 \mathrm{~min}$, which is also confirmed by larger $d R_{C} / d t$ in Fig. $5 \mathrm{~h}$. This, however, is not only a direct result of TICE on the collection kernel, which affects droplets up to a radius of $100 \mu \mathrm{m}$, but rather the effect of the increased number of large droplets produced in TICE that are able to coalesce, as expected from the larger amount of $q_{l}(20<r \leq 40 \mu \mathrm{m})$.

The variation of droplets with different sizes can also be shown by the droplet spectra of the whole cloud at $t=12,18,24$, and $30 \mathrm{~min}$, represented by the mass density distribution (Fig. 7). The difference between GRAV and TURB appears mainly in the range $r>20 \mu \mathrm{m}$, in which the collisional growth becomes important, except at $t=30 \mathrm{~min}$. At $t=30 \mathrm{~min}$, the droplet concentration decreases for $r<40 \mu \mathrm{m}$, which is a result of the collection by the settling raindrops. This decrease is larger in TURB, where precipitation is stronger. Note that the mass density of droplets larger than $r=20 \mu \mathrm{m}$ is slightly larger in TURB at $t=18 \mathrm{~min}$, while $q_{l}(20<r \leq 40 \mu \mathrm{m})$ at the locations of potential raindrops is slightly larger in GRAV (Fig. 6c). This contradiction can be explained by the fact that potential raindrops in GRAV are preferentially located in the region with larger $q_{l}(20<r \leq 40 \mu \mathrm{m})$ or, equivalently, with larger $\sigma_{r}$ as observed in Fig. 4c.

\section{c. Sensitivity to CCN concentration}

To investigate how the route to raindrop formation, discussed in the previous section, is affected by the $\mathrm{CCN}$ 


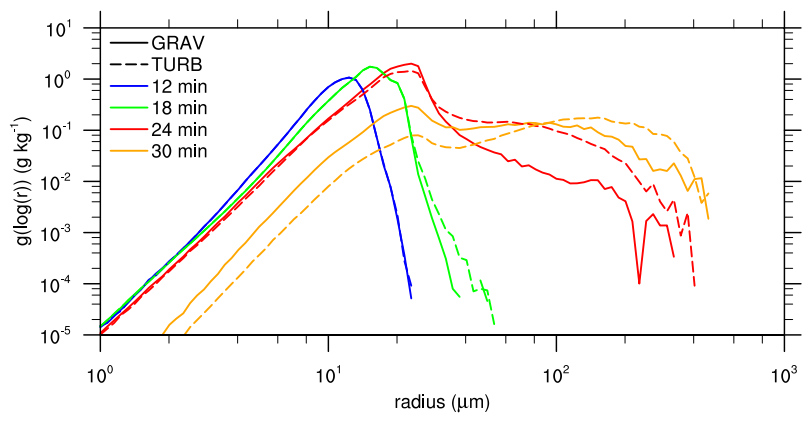

FIG. 7. Mass density distributions of droplets at $t=12,18,24$, and 30 min (solid: GRAV; dashed: TURB).

concentration, we repeated the simulation with two other initial droplet concentrations $N_{0}=20$ and $150 \mathrm{~cm}^{-3}$ in addition to the previously presented simulation with $N_{0}=70 \mathrm{~cm}^{-3}$. Figure 8 compares time series of the mass of precipitable water (the mass of all raindrops with $r>40 \mu \mathrm{m}), R, Z$, and $\varepsilon$ for the CCN concentrations of $N_{0}=20,70$, and $150 \mathrm{~cm}^{-3}$ and for GRAV and TURB.

For $N_{0}=150 \mathrm{~cm}^{-3}$, precipitation is almost inhibited since too many droplets compete for the available moisture. This delays the diffusional growth and results in smaller droplets. The delayed raindrop formation also keeps $Z$ higher in the end of the cloud life cycle, since gravitational settling is reduced. Because of increased dissipation rates at higher $\mathrm{CCN}$ concentrations (Fig. 8d), the effect of TICE becomes stronger, which increases the difference between TURB and GRAV in agreement with previous studies (Seifert et al. 2010; Benmoshe et al. 2012; Wyszogrodzki et al. 2013; Grabowski et al. 2015; Lee et al. 2015), while this difference almost vanishes for low $\mathrm{CCN}$ concentrations. It suggests that, as the diffusional growth becomes sufficiently strong at small $N_{0}$, the mechanisms to help initiate collisions, such as the broadening of DSD and TICE, are less important.

\section{d. Dependence of raindrop formation on the width of the DSD}

Figure 5 shows clearly that the broadening of DSD is necessary to trigger precipitation in GRAV but not for TURB. To understand the reason for the different dependence of DSD broadening and TICE on $\varepsilon$, we calculate the time $\tau_{R}$ required by a superdroplet to reach the size of a raindrop $(r=40 \mu \mathrm{m})$ by calculating the collisional growth of different log-normally distributed droplet spectra for different $\varepsilon$ in a simple box model. The DSD is always initialized with $q_{l}=1.0 \mathrm{~g} \mathrm{~kg}^{-1}$ but with a wide range of $\sigma_{r}$ and $r_{\text {eff }}$ of the initial DSD, resulting in about 17500 individual simulations to reach statistical convergence. The collisional growth algorithm is the same as used in the LCM, and each DSD is represented by 200 superdroplets. Note that $\tau_{R}$ is closely related to the timing of the triggering of the rapid collisional growth $T_{C}$ defined above, although they can be different sometimes. For example, $T_{C}$ may be the same for TURB and GRAV from the LCM (Fig. 8b), while $\tau_{R}$ tends to be smaller for TURB as we will show here.

Figure 9 compares the variation of $\tau_{R}$ with $\sigma_{r}$ and $r_{\text {eff }}$ under different $\varepsilon\left(\varepsilon=0\right.$ and $\left.100 \mathrm{~cm}^{2} \mathrm{~s}^{-3}\right)$. The difference of $\tau_{R}$ between $\varepsilon=0$ and $100 \mathrm{~cm}^{2} \mathrm{~s}^{-3}$ is small at large $\sigma_{r}$. However, $\tau_{R}$ increases much faster with decreasing $\sigma_{r}$ at $\varepsilon=0 \mathrm{~cm}^{2} \mathrm{~s}^{-3}$ than at $\varepsilon=100 \mathrm{~cm}^{2} \mathrm{~s}^{-3}$. It makes $\tau_{R}\left(\varepsilon=0 \mathrm{~cm}^{2} \mathrm{~s}^{-3}\right)$ much larger than $\tau_{R}\left(\varepsilon=100 \mathrm{~cm}^{2} \mathrm{~s}^{-3}\right)$ as $\sigma_{r}$ approaches zero.

Accordingly, Fig. 9 explains why the triggering of precipitation is difficult at small $\sigma_{r}$ in GRAV. If a few droplets grow by collection initially, the presence of larger droplets generated by these collections enhances the collision rate further. If the number of large droplets produced by initial collection becomes sufficiently large, it triggers the rapid collisional growth resulting in precipitation by this positive feedback, once the largest droplets reach a critical size $(r=20 \mu \mathrm{m})$. The time scale to trigger this positive feedback is characterized by $\tau_{R}$ : $\tau_{R}$ can be short under a favorable condition for collision with increased $K$, provided either by TICE or by a broad DSD; on the other hand, $\tau_{R}$ can be longer than the typical lifetime of a cloud, thus prohibiting precipitation, if the DSD is too narrow or TICE is too weak.

The fact that $\tau_{R}$ is not sensitive to $\varepsilon$ at large $\sigma_{r}$ explains why $T_{C}$ is not significantly affected by TICE, as shown in Fig. 5a. In other words, rapid collisional growth can be triggered at about the same time in both GRAV and TURB, as long as there exists a region with sufficiently large $\sigma_{r}$ in GRAV. On the other hand, in the case of TURB, the rapid collisional growth can be triggered in a region with small $\sigma_{r}$ as well. It leads to a much larger amount of precipitation in TURB, as shown in Figs. $6 \mathrm{~d}$ and 8a.

Regarding the APM simulations presented in section 3 b, it is worthwhile to mention that Fig. 9 implies that, if the APM starts with a sufficiently large $\sigma_{r}$, it can trigger the raindrop formation in time even without TICE. The assumption of a large initial $\sigma_{r}$ means, however, that the broadening of DSD is implicitly included from the start.

\section{Conclusions and discussion}

The present paper applied a recently developed Lagrangian cloud model (LCM) to clarify possible mechanisms of raindrop formation in warm clouds, which remains a key question in cloud physics. By taking advantage of the LCM, we uniquely investigated the 
(a)

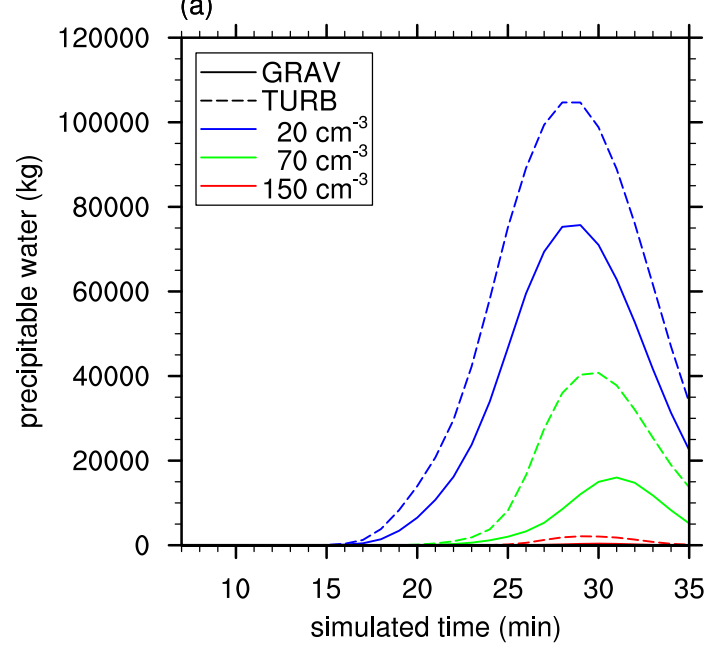

(c)

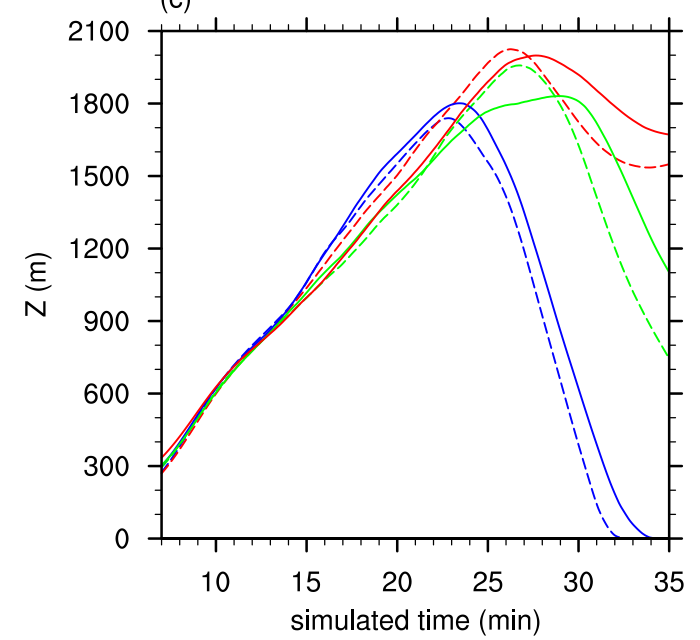

(b)

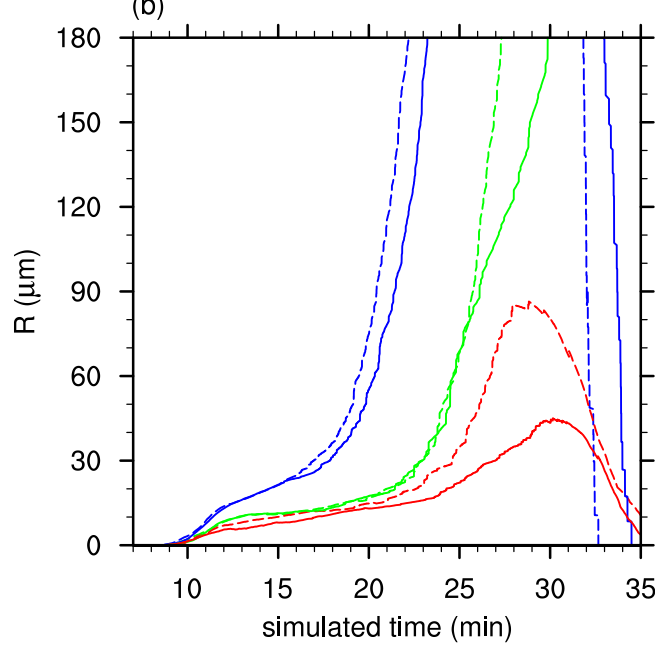

(d)

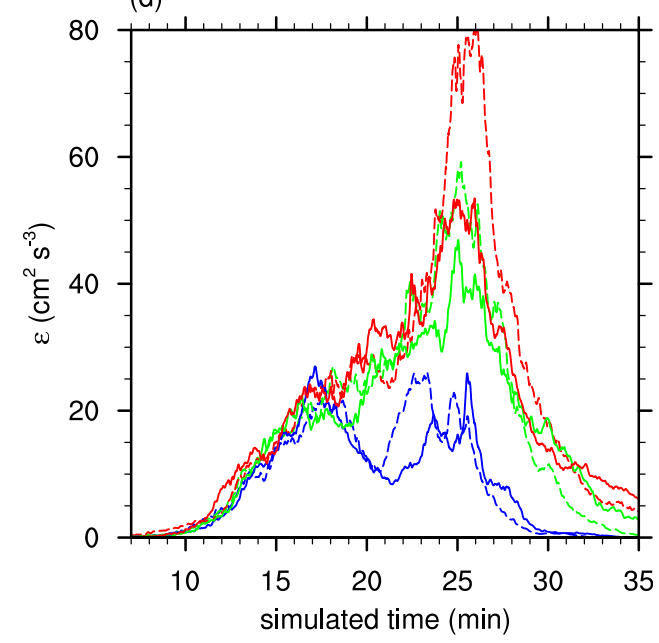

FIG. 8. Times series of variables from different initial droplet concentrations: (a) the mass of precipitable water $(r>40 \mu \mathrm{m})$, (b) $R$, (c) $Z$, and (d) $\varepsilon$ (solid: GRAV; dotted: TURB) (blue: $20 \mathrm{~cm}^{-3}$; green: $70 \mathrm{~cm}^{-3}$; red: $\left.150 \mathrm{~cm}^{-3}\right)$.

formation of raindrops directly by tracking the history of Lagrangian droplets. The present work focuses on clarifying the respective roles of different aspects of raindrop formation; that is, the broadening of the drop size distribution (DSD), which might be induced by the mixing of droplets that have experienced different supersaturation histories, and turbulence-induced collision enhancement (TICE). For this purpose, we compared the LCM results with two different collection kernels, with and without turbulence effects (TURB and GRAV, respectively), and also compared the results of the LCM with those from an adiabatic parcel model (APM), in which the broadening of the DSD due to entrainment and mixing was absent.

It is found that the rapid collisional growth, leading to the raindrop formation, is triggered when droplets with radius $r=20 \mu \mathrm{m}$ appear in the region near the cloud top, characterized by a large liquid water mixing ratio, large mean droplet size, strong turbulence, a broad DSD, and high supersaturations. The most important result is that in the shallow maritime cloud modeled here, raindrops were produced when TICE was included, regardless of the presence of any other DSD broadening mechanism, but their formation was severely delayed in the absence of TICE unless some other mechanism of DSD broadening (here, the effects of entrainment and mixing) was included. The reason leading to the difference is clarified from idealized box simulations of the collisional growth process for different DSDs. By analyzing the time to produce a raindrop $\tau_{R}(R>40 \mu \mathrm{m})$, we have found that $\tau_{R}$ is small in both GRAV and TURB at large $\sigma_{r}$, but $\tau_{R}$ increases rapidly as $\sigma_{r}$ goes to zero in GRAV, where $\sigma_{r}^{2}$ is the variance of $r$. The importance of the broadening of DSD in the raindrop formation in GRAV is also 

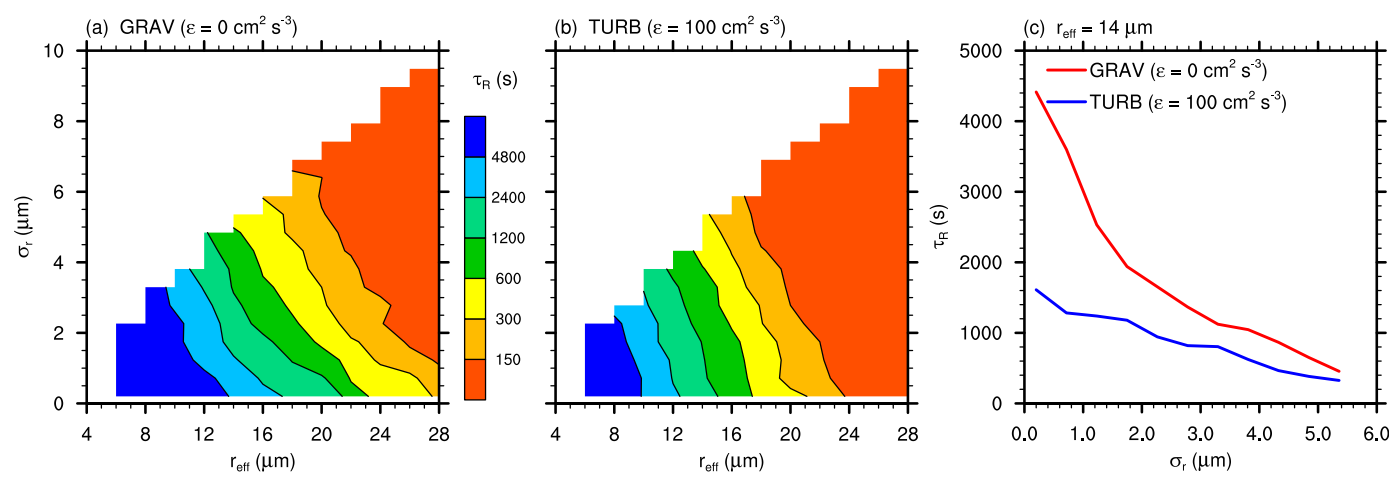

FIG. 9. The variation of the time to reach raindrops $\tau_{R}$ from box simulations of the collisional growth process starting from different log-normally shaped drop size distributions with different $\sigma_{r}$ and $r_{\text {eff }}$ : (a) GRAV $\left(\varepsilon=0 \mathrm{~cm}^{2} \mathrm{~s}^{-3}\right)$, (b) TURB $\left(\varepsilon=100 \mathrm{~cm}^{2} \mathrm{~s}^{-3}\right)$, and (c) the variation of $\tau_{R}$ with $\sigma_{r}$ for $r_{\text {eff }}=14 \mu \mathrm{m}$.

supported by the fact that the generation of raindrops is preferentially concentrated in the region of higher $\sigma_{r}$ more strongly in GRAV.

It is also found that, under the influence of TICE, the timing of the triggering of the rapid collisional growth $T_{C}$ is not significantly accelerated, but the collisional growth rate becomes much larger after $T_{C}$. It implies that the rapid collisional growth can be triggered at about the same time in GRAV and TURB, as long as there exists a region with sufficiently large $\sigma_{r}$ in GRAV. On the other hand, in the case of TURB, the rapid collisional growth can be triggered in a region with small $\sigma_{r}$ as well, which leads to a much larger amount of precipitation in TURB. Simulations with different CCN concentrations show that higher droplet concentrations increase the time for raindrop formation and decrease precipitation, as expected, but intensify the effect of TICE in agreement with previous studies.

Furthermore, the results demonstrate how significantly entrainment and mixing affect the simulated cloud; especially strong turbulence as a result of evaporative cooling as well as the large variability of the supersaturation within the cloud, including subsaturated regions, are necessary for TICE and the broadening of the DSD, respectively. It is important to note, however, that both TICE and DSD broadening are fundamentally linked to turbulence, ranging from convection and entrainment to small-scale turbulence on the droplet scale. The present results suggest though, if turbulence within the cloud is sufficiently strong, the rapid collisional growth leading to precipitation is triggered regardless of the broadening of DSD. On the other hand, the broadening of DSD can play an important role in a weakly turbulent cloud. The intensity of turbulence varies widely depending on the cloud type; for example, dissipation rates of $1-10 \mathrm{~cm}^{2} \mathrm{~s}^{-3}$ have been observed in stratocumulus, $10-100 \mathrm{~cm}^{2} \mathrm{~s}^{-3}$ in shallow convective clouds, and values as large as $1000 \mathrm{~cm}^{2} \mathrm{~s}^{-3}$ in deep convective clouds (e.g., Pruppacher and Klett 1997; Siebert et al. 2006; Shupe et al. 2012).

There are many areas where future work is yet required. The uncertainty of the existing collection kernels is still large, not only in the collection kernel including turbulence effects but also in the gravitational collection kernel itself (Klett and Davis 1973; Khain et al. 2007). Furthermore, the present simulation assumes homogeneous mixing within the grid, similar to many other cloud models. However, inhomogeneous mixing can make the DSD broadening larger than in the present simulations (Lasher-Trapp et al. 2005). Other DSD broadening mechanisms need to be addressed in future studies, too: (subgrid scale) eddy hopping (Grabowski and Abade 2017), the activation characteristics of the underlying aerosol distribution (Hudson and Yum 1997), or the presence of giant and ultragiant aerosols (Johnson 1982).

Acknowledgments. This work has been funded by the Korea Meteorological Administration Research and Development Program under Grant KMIPA 2015-5180 and the German Research Foundation (DFG) under Grant RA 617/27-1. This LES/LCM model used in this study (revision 1891) is publicly available (https://palm. muk.uni-hannover.de/trac/browser/palm?rev $=1891$ ). For analysis, the model has been extended and additional analysis tools have been developed. The code is available from the authors on request. Most of the simulations have been carried out on the Cray XC-40 systems of the North-German Supercomputing Alliance (HLRN). The Supercomputing Center/Korea Institute of Science and Technology Information (KISTI; KSC-2015-C2-042) has been also used for analysis. The authors thank L. P. Wang for his suggestions on the collision algorithm and three anonymous reviewers for their helpful comments on the manuscript. 
(a)

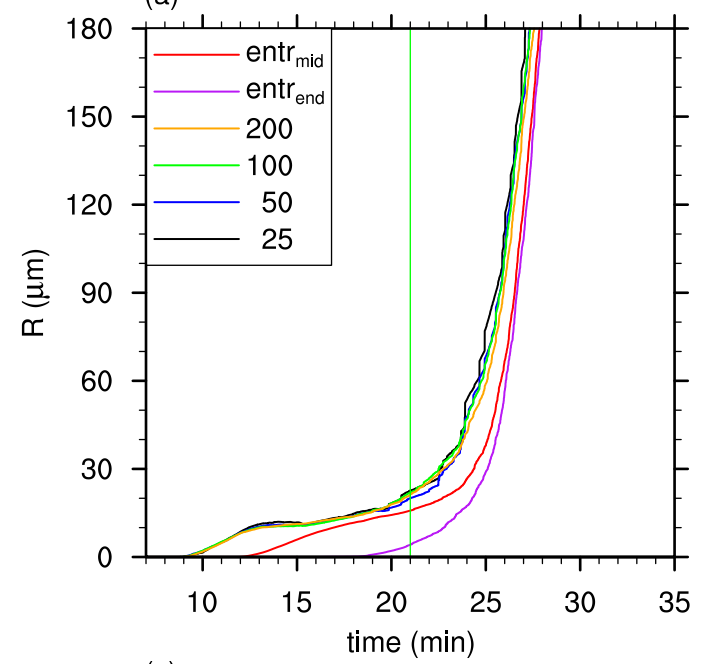

(c)

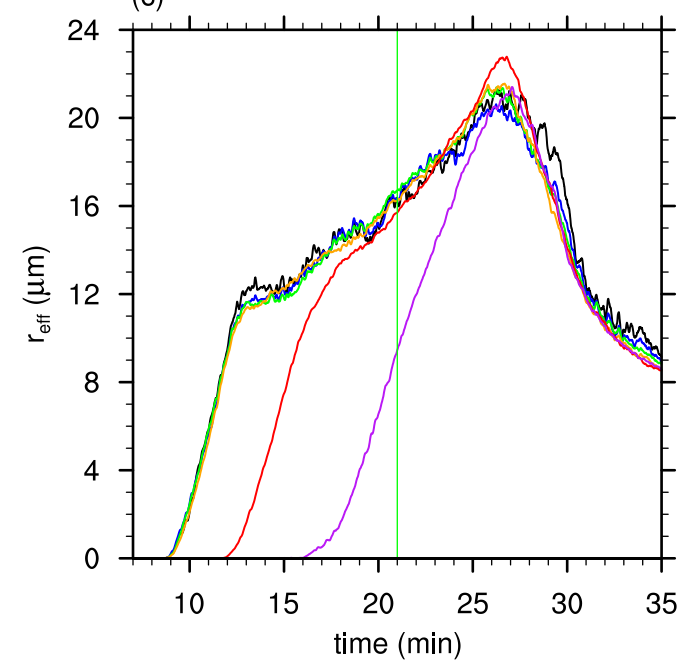

(b)

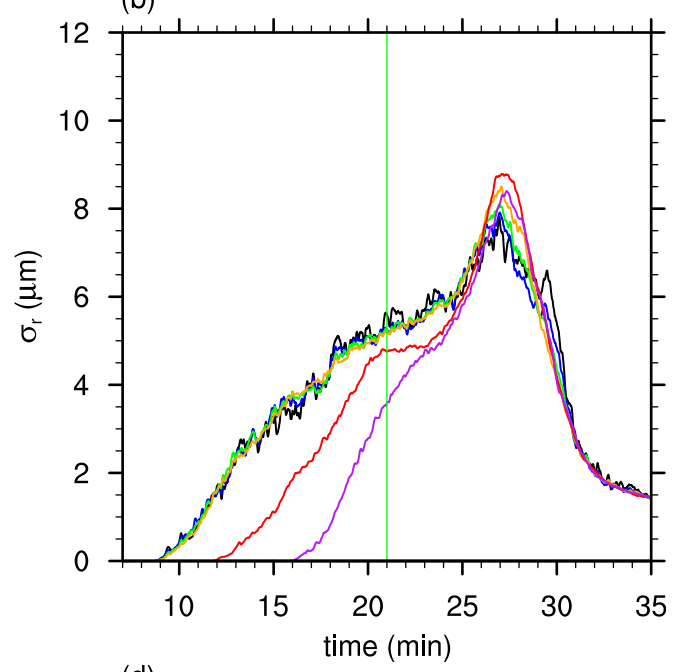

(d)

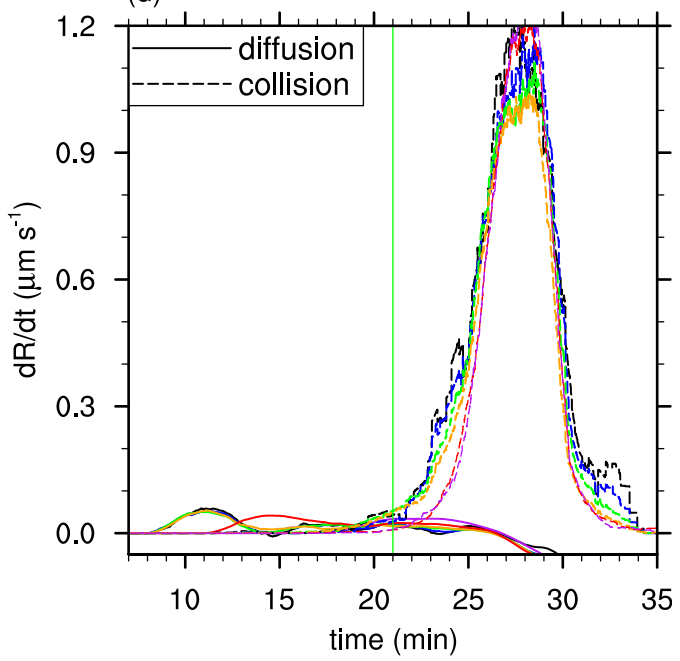

FIG. B1. Time series of the ensemble average of physical variables following potential raindrops in TURB with different sampling sizes and without filtering [black: 25; blue: 50; green: 100; yellow: 200; red: entrained between 12 and $16 \mathrm{~min}$ (entr $\mathrm{mid}_{\text {mid }}$; purple: entrained after $\left.16 \mathrm{~min}\left(\mathrm{entr}_{\mathrm{end}}\right)\right]$ : (a) $R$, (b) $\sigma_{r}$, (c) $r_{\text {eff }}$, and (d) $d R_{C} / d t$ and $d R_{D} / d t$.

\section{APPENDIX A}

\section{The Adiabatic Parcel Model}

In this study, an adiabatic parcel model (APM) is used to calculate adiabatic values of quantities along the trajectories of individual particles that have been calculated and tracked in the Lagrangian cloud model (LCM). The APM is based on the prognostic equations for the evolution of the supersaturation,

$$
\frac{d S}{d t}=\left(\frac{g L_{v}}{c_{p} R_{v} T^{2}}-\frac{g}{R_{a} T}\right) \frac{d Z}{d t}-\left(\frac{1}{q}-\frac{L_{v}^{2}}{c_{p} R_{v} T^{2}}\right)\left(\frac{d q_{l}}{d t}\right)_{\text {cond }},
$$

and potential temperature,

$$
\frac{d \theta}{d t}=\frac{L_{v}}{\Pi c_{p}}\left(\frac{d q_{l}}{d t}\right)_{\text {cond }},
$$

in an adiabatically lifted parcel (e.g., Korolev and Mazin 2003). These equations are driven by the vertical motion of the parcel, $d Z / d t$, and the condensation or evaporation of liquid water, $\left(d q_{l} / d t\right)_{\text {cond }}$. Furthermore, changes in the water vapor mixing ratio are directly linked to changes in the liquid water mixing ratio $q$ :

$$
\left(\frac{d q}{d t}\right)_{\text {cond }}=-\left(\frac{d q_{l}}{d t}\right)_{\text {cond }},
$$

which are calculated as in the LCM by the diffusional growth equation [see (8)], using the adiabatic supersaturation and temperature from (A1) and (A2), 
respectively. The adiabatic parcel is assumed to have the same properties as one grid cell in the LCM simulations-that is, a volume of $20 \times 20 \times 20 \mathrm{~m}^{3}$ and 200 superdroplets from which the cloud microphysics are calculated by (8) for diffusional and (12)(14) for collisional growth.

The most important information taken from the tracked particles is their height $Z$. Furthermore, $S, \theta$, and $q$ in (A1)-(A3) are initialized by their respective values of the particle trajectory as soon as the particle is moved into a supersaturated grid cell. The adiabatic DSD is disturbed to produce a small, but finite width of DSD $\left(\sigma_{r}=0.05 \mu \mathrm{m}\right)$, as soon as the droplets grow larger than $r=10 \mu \mathrm{m}$. Equations (A1)-(A3) are then integrated using the same time step as the LCM. In the case of TURB, the dissipation rate is also taken from the tracked droplets' trajectories to steer turbulenceenhanced collisions.

Note that the dynamics of the APM are driven by the LCM, but thermodynamics and cloud microphysics are independent. In that sense, the chosen approach resembles the piggybacking method of Grabowski (2015), which allows a direct assessment of the APM cloud microphysics driven by the same dynamics as simulated in the LCM - that is, in the identical dynamical framework without affecting it.

\section{APPENDIX B}

\section{Sensitivity to the Sampling of Potential Raindrops}

The analysis of this study is based on following individual potential raindrops, which are defined as the Lagrangian superdroplets whose maximum size during the evolution of the cloud belongs to the largest 50 droplets. The sampling is based on the fact that only 251 and 2982 superdroplets in GRAV and TURB, respectively, grow larger than $250 \mu \mathrm{m}$ in radius during this period, and from these superdroplets only 51 and 211 superdroplets are entrained into the cloud before $t=$ $12 \mathrm{~min}$. Therefore, we chose the largest 50 superdroplets for both GRAV and TURB for analysis. The growth of superdroplets that are entrained after $t=12 \mathrm{~min}$ starts later and, therefore, shifts the starting point of the time series to the later time. These superdroplets are therefore filtered out in Fig. 5 in order to focus on the distinct features of droplets with similar entrainment times.

Nonetheless, it is necessary to examine how the time series are modified if all superdroplets are considered without filtering. Figure B1 compares the time series of $R, \sigma_{r}, r_{\text {eff }}, d R_{C} / d t$, and $d R_{D} / d t$ corresponding to Fig. 5 from the data with different sampling sizes (the largest
$25,50,100$, and 200 superdroplets entrained before $t=$ $12 \mathrm{~min})$ and different entrainment times $[12<t<$ $\left.16 \mathrm{~min}\left(\mathrm{entr}_{\mathrm{mid}}\right), 16 \mathrm{~min}<t\left(\mathrm{entr}_{\mathrm{end}}\right)\right]$. The time series show that they are insensitive to the sampling size, thus indicating the robustness of the results shown in Fig. 5.

Meanwhile, the superdroplets that are entrained to the cloud later delay the growth of $R, \sigma_{r}, r_{\mathrm{eff}}, d R_{C} / d t$, and $d R_{D} / d t$, since the starting time of the droplet growth is shifted to a later time. However, the timing of the rapid collisional growth $T_{C}$ is only slightly delayed ( $\approx 2 \mathrm{~min}$ ), much smaller than the difference in the starting time of diffusional growth, and the differences in the variables almost disappear at $t=27 \mathrm{~min}$. The main reason is that the start of the collisional growth of the superdroplets entrained earlier $(t<12 \mathrm{~min})$ already increased the values of $\sigma_{r}$ and $r_{\text {eff }}$ inside the cloud, which provides the favorable background condition for the collisional growth of the superdroplets entrained later $(t>12 \mathrm{~min})$. It is also found that the rapid collisional growth is triggered when $R$ reaches $20 \mu \mathrm{m}$, regardless of entrainment times.

\section{REFERENCES}

Andrejczuk, M., J. M. Reisner, B. Henson, M. K. Dubey, and C. A. Jeffery, 2008: The potential impacts of pollution on a nondrizzling stratus deck: Does aerosol number matter more than type? J. Geophys. Res., 113, D19204, doi:10.1029/ 2007JD009445.

- W. W. Grabowski, J. M. Reisner, and A. Gadian, 2010: Cloud-aerosol interactions for boundary layer stratocumulus in the Lagrangian Cloud Model. J. Geophys. Res., 115, D22214, doi:10.1029/2010JD014248.

Arabas, S., and S.-I. Shima, 2013: Large-eddy simulations of trade wind cumuli using particle-based microphysics with Monte Carlo coalescence. J. Atmos. Sci., 70, 2768-2777, doi:10.1175/ JAS-D-12-0295.1.

- H. Pawlowska, and W. Grabowski, 2009: Effective radius and droplet spectral width from in-situ aircraft observations in trade-wind cumuli during RICO. Geophys. Res. Lett., 36, L11803, doi:10.1029/2009GL038257.

Ayala, O., B. Rosa, and L.-P. Wang, 2008: Effects of turbulence on the geometric collision rate of sedimenting droplets. Part 2. Theory and parameterization. New J. Phys., 10, 075016, doi:10.1088/1367-2630/10/7/075016.

Baker, M. B., R. G. Corbin, and J. Latham, 1980: The influence of entrainment on the evolution of cloud droplet spectra: I. A model of inhomogeneous mixing. Quart. J. Roy. Meteor. Soc., 106, 581-598, doi:10.1002/qj.49710644914.

Bannon, P. R., 2002: Theoretical foundations for models of moist convection. J. Atmos. Sci., 59, 1967-1982, doi:10.1175/ 1520-0469(2002)059<1967:TFFMOM>2.0.CO;2.

Beard, K. V., and H. T. Ochs III, 1984: Collection and coalescence efficiencies for accretion. J. Geophys. Res., 89, 7165-7169, doi:10.1029/JD089iD05p07165.

$\longrightarrow$, and - , 1993: Warm-rain initiation: An overview of microphysical mechanisms. J. Appl. Meteor., 32, 608-625, doi:10.1175/1520-0450(1993)032<0608:WRIAOO>2.0.CO;2. 
Benmoshe, N., M. Pinsky, A. Pokrovsky, and A. Khain, 2012: Turbulent effects on the microphysics and initiation of warm rain in deep convective clouds: $2-\mathrm{D}$ simulations by a spectral mixed-phase microphysics cloud model. J. Geophys. Res., 117, D06220, doi:10.1029/2011JD016603.

Berry, E. X., and R. L. Reinhardt, 1974: An analysis of cloud drop growth by collection: Part II. Single initial distributions. J. Atmos. Sci, 31, 1825-1831, doi:10.1175/1520-0469(1974)031<1825: $\mathrm{AAOCDG}>2.0 . \mathrm{CO} ; 2$.

Blyth, A. M., 1993: Entrainment in cumulus clouds. J. Appl. Meteor., 32, 626-640, doi:10.1175/1520-0450(1993)032<0626: EICC $>2.0 . \mathrm{CO} ; 2$.

Cooper, W. A., 1989: Effects of variable droplet growth histories on droplet size distributions. Part I: Theory. J. Atmos. Sci., 46, 1301-1311, doi:10.1175/1520-0469(1989)046<1301: EOVDGH $>2.0 . \mathrm{CO} ; 2$.

— S. G. Lasher-Trapp, and A. M. Blyth, 2013: The influence of entrainment and mixing on the initial formation of rain in a warm cumulus cloud. J. Atmos. Sci., 70, 1727-1743, doi:10.1175/JAS-D-12-0128.1.

Devenish, B. J., and Coauthors, 2012: Droplet growth in warm turbulent clouds. Quart. J. Roy. Meteor. Soc., 138, 1401-1429, doi:10.1002/qj.1897.

Franklin, C. N., 2008: A warm rain microphysics parameterization that includes the effect of turbulence. J. Atmos. Sci., 65, 17951816, doi:10.1175/2007JAS2556.1.

Freud, E., and D. Rosenfeld, 2012: Linear relation between convective cloud drop number concentration and depth for rain initiation. J. Geophys. Res., 117, D02207, doi:10.1029/2011JD016457.

Gerber, H. E., G. M. Frick, J. B. Jensen, and J. G. Hudson, 2008: Entrainment, mixing, and microphysics in trade-wind cumulus. J. Meteor. Soc. Japan, 86A, 87-106, doi:10.2151/ jmsj.86A.87.

Grabowski, W. W., 2015: Untangling microphysical impacts on deep convection applying a novel modeling methodology. J. Atmos. Sci., 72, 2446-2464, doi:10.1175/JAS-D-14-0307.1.

- , and L.-P. Wang, 2013: Growth of cloud droplets in a turbulent environment. Annu. Rev. Fluid Mech., 45, 293-324, doi:10.1146/annurev-fluid-011212-140750.

_- , and G. C. Abade, 2017: Broadening of cloud droplet spectra through eddy hopping: Turbulent adiabatic parcel simulations. J. Atmos. Sci., 74, 1485-1493, doi:10.1175/ JAS-D-17-0043.1.

— , L.-P. Wang, and T. V. Prabha, 2015: Macroscopic impacts of cloud and precipitation processes on maritime shallow convection as simulated by a large eddy simulation model with bin microphysics. Atmos. Chem. Phys., 15, 913-926, doi:10.5194/ acp-15-913-2015.

Hall, W. D., 1980: A detailed microphysical model within a twodimensional dynamic framework: Model description and preliminary results. J. Atmos. Sci., 37, 2486-2507, doi:10.1175/ 1520-0469(1980)037<2486:ADMMWA > 2.0.CO;2.

Hoffmann, F., 2016: The effect of spurious cloud edge supersaturations in Lagrangian cloud models: An analytical and numerical study. Mon. Wea. Rev., 144, 107-118, doi:10.1175/ MWR-D-15-0234.1.

— S. Raasch, and Y. Noh, 2015: Entrainment of aerosols and their activation in a shallow cumulus cloud studied with a coupled LCM-LES approach. Atmos. Res., 156, 43-57, doi:10.1016/j.atmosres.2014.12.008.

Hudson, J. G., and S. S. Yum, 1997: Droplet spectral broadening in marine stratus. J. Atmos. Sci., 54, 2642-2654, doi:10.1175/ 1520-0469(1997)054<2642:DSBIMS >2.0.CO;2.
Jensen, J. B., and S. Lee, 2008: Giant sea-salt aerosols and warm rain formation in marine stratocumulus. J. Atmos. Sci., 65, 3678-3694, doi:10.1175/2008JAS2617.1.

Johnson, D. B., 1982: The role of giant and ultragiant aerosol particles in warm rain initiation. J. Atmos. Sci., 39, 448-460, doi:10.1175/1520-0469(1982)039<0448:TROGAU>2.0.CO;2.

Kessler, E., 1969: On the Distribution and Continuity of Water Substance in Atmospheric Circulations. Meteor. Monogr., No. 32, Amer. Meteor. Soc., 84 pp.

Khain, A., M. Pinsky, T. Elperin, N. Kleeorin, I. Rogachevskii, and A. Kostinski, 2007: Critical comments to results of investigations of drop collisions in turbulent clouds. Atmos. Res., 86, 1-20, doi:10.1016/j.atmosres.2007.05.003.

-, T. V. Prabha, N. Benmoshe, G. Pandithurai, and M. Ovchinnikov, 2013: The mechanism of first raindrops formation in deep convective clouds. J. Geophys. Res. Atmos., 118, 9123-9140, doi:10.1002/jgrd.50641.

Klett, J., and M. Davis, 1973: Theoretical collision efficiencies of cloud droplets at small Reynolds numbers. J. Atmos. Sci., 30, 107-117, doi:10.1175/1520-0469(1973)030<0107: TCEOCD $>2.0 . \mathrm{CO} ; 2$.

Kogan, Y., 2013: A cumulus cloud microphysics parameterization for cloud-resolving models. J. Atmos. Sci., 70, 1423-1436, doi:10.1175/JAS-D-12-0183.1.

Korolev, A. V., and I. P. Mazin, 2003: Supersaturation of water vapor in clouds. J. Atmos. Sci., 60, 2957-2974, doi:10.1175/ 1520-0469(2003)060<2957:SOWVIC $>2.0 . C O ; 2$.

Lasher-Trapp, S. G., C. A. Knight, and J. M. Straka, 2001: Early radar echoes from ultragiant aerosol in a cumulus congestus: Modeling and observations. J. Atmos. Sci., 58, 3545-3562, doi:10.1175/1520-0469(2001)058<3545: EREFUA $>2.0 . \mathrm{CO} ; 2$.

- W. A. Cooper, and A. M. Blyth, 2005: Broadening of droplet size distributions from entrainment and mixing in a cumulus cloud. Quart. J. Roy. Meteor. Soc., 131, 195-220, doi:10.1256/ qj.03.199.

Lee, H., J.-J. Baik, and J.-Y. Han, 2015: Effects of turbulence on warm clouds and precipitation with various aerosol concentrations. Atmos. Res., 153, 19-33, doi:10.1016/j.atmosres.2014.07.026.

Lee, J., Y. Noh, S. Raasch, T. Riechelmann, and L.-P. Wang, 2014: Investigation of droplet dynamics in a convective cloud using a Lagrangian cloud model. Meteor. Atmos. Phys., 124, 1-21, doi:10.1007/s00703-014-0311-y.

Maronga, B., and Coauthors, 2015: The Parallelized Large-Eddy Simulation Model (PALM) version 4.0 for atmospheric and oceanic flows: Model formulation, recent developments, and future perspectives. Geosci. Model Dev., 8, 2515-2551, doi:10.5194/gmd-8-2515-2015.

Naumann, A. K., and A. Seifert, 2015: A Lagrangian drop model to study warm rain microphysical processes in shallow cumulus. J. Adv. Model. Earth Syst., 7, 1136-1154, doi:10.1002/ 2015MS000456.

Pinsky, M., and A. Khain, 2002: Effects of in-cloud nucleation and turbulence on droplet spectrum formation in cumulus clouds. Quart. J. Roy. Meteor. Soc., 128, 501-533, doi:10.1256/ 003590002321042072.

Politovich, M. K., and W. A. Cooper, 1988: Variability of the supersaturation in cumulus clouds. J. Atmos. Sci., 45, 1651-1664, doi:10.1175/1520-0469(1988)045<1651:VOTSIC >2.0.CO;2.

Pruppacher, R. H., and J. D. Klett, 1997: Microphysics of Clouds and Precipitation. 2nd ed. Atmospheric and Oceanographic Sciences Library, Vol. 18, Kluwer Academic Publishers, 954 pp., doi:10.1007/978-0-306-48100-0. 
Raasch, S., and M. Schröter, 2001: PALM-A large-eddy simulation model performing on massively parallel computers. $M e$ teor. Z., 10, 363-372, doi:10.1127/0941-2948/2001/0010-0363.

Rauber, R. M., and Coauthors, 2007: Rain in Shallow Cumulus over the Ocean: The RICO campaign. Bull. Amer. Meteor. Soc., 88, 1912-1928, doi:10.1175/BAMS-88-12-1912.

Riechelmann, T., Y. Noh, and S. Raasch, 2012: A new method for large-eddy simulations of clouds with Lagrangian droplets including the effects of turbulent collision. New J. Phys., 14, 065008, doi:10.1088/1367-2630/14/6/065008.

—, U. Wacker, K. D. Beheng, D. Etling, and S. Raasch, 2015: Influence of turbulence on the drop growth in warm clouds, Part II: Sensitivity studies with a spectral bin microphysics and a Lagrangian cloud model. Meteor. Z., 24, 293-311, doi:10.1127/metz/2015/0608.

Rogers, R. R., and M. K. Yau, 1989: A Short Course in Cloud Physics. 3rd ed. International Series in Natural Philosophy, Pergamon Press, 293 pp.

, D. Baumgardner, S. A. Ethier, D. A. Carter, and W. L. Ecklund, 1993: Comparison of raindrop size distributions measured by radar wind profiler and by airplane. J. Appl. Meteor., 32, 694-699, doi:10.1175/1520-0450(1993)032<0694: CORSDM $>2.0 . \mathrm{CO} ; 2$.

Seifert, A., L. Nuijens, and B. Stevens, 2010: Turbulence effects on warm-rain autoconversion in precipitating shallow convection. Quart. J. Roy. Meteor. Soc., 136, 1753-1762, doi:10.1002/ qj.684.

Shaw, R. A., 2003: Particle-turbulence interactions in atmospheric clouds. Annu. Rev. Fluid Mech., 35, 183-227, doi:10.1146/ annurev.fluid.35.101101.161125.

Shima, S.-I., K. Kusano, A. Kawano, T. Sugiyama, and S. Kawahara, 2009: The super-droplet method for the numerical simulation of clouds and precipitation: A particlebased and probabilistic microphysics model coupled with a non-hydrostatic model. Quart. J. Roy. Meteor. Soc., 135, 13071320, doi:10.1002/qj.441.

Shupe, M., I. Brooks, and G. Canut, 2012: Evaluation of turbulent dissipation rate retrievals from Doppler cloud radar. Atmos. Meas. Tech., 5, 1375-1385, doi:10.5194/amt-5-1375-2012.

Siebert, H., K. Lehmann, and M. Wendisch, 2006: Observations of small-scale turbulence and energy dissipation rates in the cloudy boundary layer. J. Atmos. Sci., 63, 1451-1466, doi:10.1175/JAS3687.1.

Small, J. D., and P. Y. Chuang, 2008: New observations of precipitation initiation in warm cumulus clouds. J. Atmos. Sci., 65 , 2972-2982, doi:10.1175/2008JAS2600.1.

Sölch, I., and B. Kärcher, 2010: A large-eddy model for cirrus clouds with explicit aerosol and ice microphysics and Lagrangian ice particle tracking. Quart. J. Roy. Meteor. Soc., 136, 2074-2093, doi:10.1002/qj.689.

Telford, J., 1955: A new aspect of coalescence theory. J. Meteor., 12, 436-444, doi:10.1175/1520-0469(1955)012<0436: ANAOCT $>2.0 . C O ; 2$.

Unterstrasser, S., F. Hoffmann, and M. Lerch, 2017: Collection/ aggregation algorithms in Lagrangian cloud microphysical models: Rigorous evaluation in box model simulations. Geosci. Model Dev., 10, 1521-1548, doi:10.5194/gmd-10-1521-2017.

vanZanten, M. C., and Coauthors, 2011: Controls on precipitation and cloudiness in simulations of trade-wind cumulus as observed during RICO. J. Adv. Model. Earth Syst., 3, M06001, doi:10.1029/2011MS000056.

Wang, L.-P., and W. W. Grabowski, 2009: The role of air turbulence in warm rain initiation. Atmos. Sci. Lett., 10, 1-8, doi:10.1002/asl.210.

Wyszogrodzki, A. A., W. W. Grabowski, L.-P. Wang, and O. Ayala, 2013: Turbulent collision-coalescence in maritime shallow convection. Atmos. Chem. Phys., 13, 8471-8487, doi:10.5194/acp-13-8471-2013. 